\title{
$h p$-Adaptive Discontinuous Galerkin Finite Element Methods for First-Order Hyperbolic Problems
}

\author{
Paul Houston ${ }^{1}$ and Endre Süli ${ }^{2}$
}

\begin{abstract}
We consider the a posteriori error analysis of $h p$-discontinuous Galerkin finite element approximations to first-order hyperbolic problems. In particular, we discuss the question of error estimation for linear functionals, such as the outflow flux and the local average of the solution. Based on our a posteriori error bound we design and implement the corresponding adaptive algorithm to ensure reliable and efficient control of the error in the prescribed functional to within a given tolerance. This involves exploiting both local polynomial-degree-variation and local mesh subdivision. The theoretical results are illustrated by a series of numerical experiments.
\end{abstract}

Subject classification: AMS(MOS) 65N12, 65N15, 65N30

Keywords: $h p$-finite element methods, a posteriori error analysis, hyperbolic problems

Abbreviated title: $h p$-adaptive DGFEM for hyperbolic problems

\section{Introduction}

Adaptive finite element methods that are capable of exploiting both local polynomial degree variation ( $p$-refinement) and local mesh subdivision ( $h$-refinement) offer greater flexibility and improved efficiency than mesh refinement methods which only incorporate $h$-refinement or $p$-refinement in isolation. The aim of this paper is to develop the a posteriori error analysis of the $h p$-version of the discontinuous Galerkin finite element method; see [8] and the references cited therein, and [6] for earlier work in this area. In particular, we shall be concerned with the derivation of computable error bounds for linear functionals of the solution to scalar first-order hyperbolic problems. Relevant examples of linear functionals of the solution include the mean value of the field over the computational domain and the normal flux through the outflow boundary.

The paper is structured as follows. In Section 2 we introduce the model problem and formulate its discontinuous Galerkin finite element approximation. Then, in

\footnotetext{
${ }^{1}$ Department of Mathematics and Computer Science, University of Leicester, University Road, Leicester LE1 7RH, UK

${ }^{2}$ University of Oxford, Computing Laboratory, Wolfson Building, Parks Road, Oxford OX1 3QD, UK.
} 
Section 3, we derive both a posteriori and a priori error bounds for linear functionals of the solution. Our a posteriori error bounds stem from a hyperbolic duality argument and include computable residual terms multiplied by local weights involving the dual solution, cf. [12, 16]. Guided by our a posteriori error analysis, in Section 4 we design an $h p$-adaptive finite element algorithm to guarantee both reliable and efficient control of the error in the computed functional with respect to a fixed user-defined tolerance. A key question in $h p$-adaptive algorithms is how to automatically decide when to $h$-refine/derefine and when to $p$-refine/derefine. Here, stimulated by the work of Ainsworth and Senior [2], we develop a decision mechanism based on the estimation of local Sobolev regularities of the primal and dual solutions by means of the a priori error bounds from Section 3 (see also [20]). The performance of the resulting $h p$-refinement strategy is then studied in Section 5 through a series of numerical experiments. In particular, we demonstrate the superiority of using $h p$-adaptive mesh refinement over the traditional $h$-refinement method, where the degree of the approximating polynomial is kept fixed at some low value. Finally, in Section 6 we summarise the work presented in this paper and draw some conclusions.

\section{Model problem and discretisation}

Let $\Omega$ be a bounded open polyhedral domain in $\mathbb{R}^{d}, d \geq 2$, and let $\Gamma$ denote the union of open faces of $\Omega$. Suppose that $\mathbf{b}=\left(b_{1}, \ldots, b_{d}\right)$ is a $d$-component vector function defined on $\bar{\Omega}$ with $b_{i} \in W_{\infty}^{1}(\Omega), i=1, \ldots, d$, and consider the following subsets of $\Gamma$ :

$$
\Gamma_{-}=\{x \in \Gamma: \mathbf{b}(x) \cdot \mathbf{n}(x)<0\}, \quad \Gamma_{+}=\{x \in \Gamma: \mathbf{b}(x) \cdot \mathbf{n}(x)>0\} ;
$$

here, $\mathbf{n}(x)$ denotes the unit outward normal vector to $\Gamma$ at $x \in \Gamma$. The sets $\Gamma_{-}$and $\Gamma_{+}$are referred to as the inflow and outflow boundary, respectively. We shall suppose that $\Gamma$ is almost everywhere non-characteristic in the sense that the set $\Gamma \backslash\left(\Gamma_{-} \cup \Gamma_{+}\right)$ has $(d-1)$-dimensional measure zero. Given $c \in L_{\infty}(\Omega), f \in L_{2}(\Omega)$ and $g \in L_{2}\left(\Gamma_{-}\right)$, we consider the following boundary-value problem: find $u \in H(\mathcal{L}, \Omega)$ such that

$$
\begin{aligned}
\mathcal{L} u \equiv \mathbf{b} \cdot \nabla u+c u & =f, \quad x \in \Omega, \\
u & =g, \quad x \in \Gamma_{-},
\end{aligned}
$$

where $H(\mathcal{L}, \Omega)=\left\{v \in L_{2}(\Omega): \mathcal{L} v \in L_{2}(\Omega)\right\}$ denotes the graph space of the partial differential operator $\mathcal{L}$ in $L_{2}(\Omega)$. In addition, we adopt the following (standard) hypothesis: there exists a constant vector $\xi \in \mathbb{R}^{d}$ and a positive real number $\delta$ such that

$$
c-\frac{1}{2} \nabla \cdot \mathbf{b}+\mathbf{b} \cdot \xi \geq \delta^{2} \text { for a.e. } x \in \Omega .
$$

We note that assumption (2.2) ensures the existence of a unique solution $u \in$ $H(\mathcal{L}, \Omega)$ to $(2.1)$, cf. [13], for example. General results concerning the existence 
and uniqueness of solutions to boundary value problems for first-order hyperbolic equations are given in [4], and [9] pp. 215-262. For the sake of simplicity of presentation, we assume that $(2.2)$ is satisfied with $\xi=0$, and we define $c_{0} \in L_{\infty}(\Omega)$ by

$$
c_{0}^{2}(x)=c(x)-\frac{1}{2} \nabla \cdot \mathbf{b}(x), \quad x \in \Omega .
$$

Clearly, $c_{0}(x) \geq \delta>0$ for almost every $x \in \Omega$.

\section{$2.1 \quad$ Finite element spaces}

Suppose that $\mathcal{T}$ is a regular or 1-irregular subdivision of $\Omega$ into disjoint open element domains $\kappa$ such that $\bar{\Omega}=\cup_{\kappa \in \mathcal{T}} \bar{\kappa}$. Thus a $(d-1)$-dimensional face of each element $\kappa$ in $\mathcal{T}$ is allowed to contain at most one hanging (irregular) node - typically the barycenter of the face. It will be assumed that $\mathcal{T}$ respects the decomposition of $\Gamma$ into $\Gamma_{-}$and $\Gamma_{+}$in the following sense: if a $(d-1)$-dimensional open face $e$ of an element $\kappa$ lies on $\Gamma$ then $e$ is a subset of either $\Gamma_{-}$or $\Gamma_{+}$. We shall suppose that the family of subdivisions $\mathcal{T}$ is shape-regular (cf. pp. 61 and 113 and Remark 2.2 on p. 114 in [7], for example) and that each $\kappa \in \mathcal{T}$ is a smooth bijective image of a fixed master element $\hat{\kappa}$, that is, $\kappa=F_{\kappa}(\hat{\kappa})$ for all $\kappa \in \mathcal{T}$ where $\hat{\kappa}$ is either the open unit simplex or the open unit hypercube in $\mathbb{R}^{d}$. On the reference element $\hat{\kappa}$ we define spaces of polynomials of degree $p \geq 1$ as follows:

$$
\mathcal{Q}_{p}=\operatorname{span}\left\{\hat{x}^{\alpha}: 0 \leq \alpha_{i} \leq p, 1 \leq i \leq d\right\}, \quad \mathcal{P}_{p}=\operatorname{span}\left\{\hat{x}^{\alpha}: 0 \leq|\alpha| \leq p\right\} .
$$

To each $\kappa \in \mathcal{T}$ we assign an integer $p_{\kappa} \geq 1$; collecting the $p_{\kappa}$ and $F_{\kappa}$ in the vectors $\mathbf{p}=\left\{p_{\kappa}: \kappa \in \mathcal{T}\right\}$ and $\mathbf{F}=\left\{F_{\kappa}: \kappa \in \mathcal{T}\right\}$, respectively, we introduce the finite element space

$S^{\mathbf{p}}(\Omega, \mathcal{T}, \mathbf{F})=\left\{u \in L_{2}(\Omega):\left.u\right|_{\kappa} \circ F_{\kappa} \in \mathcal{Q}_{p_{\kappa}}\right.$ if $F_{\kappa}^{-1}(\kappa)$ is the open unit hypercube, and $\left.u\right|_{\kappa} \circ F_{\kappa} \in \mathcal{P}_{p_{\kappa}}$ if $F_{\kappa}^{-1}(\kappa)$ is the open unit simplex; $\left.\kappa \in \mathcal{T}\right\}$.

Assuming that $\mathcal{T}$ is a subdivision of $\Omega$, we consider the broken Sobolev space $H^{\mathrm{s}}(\Omega, \mathcal{T})$ of composite index $\mathrm{s}$ with nonnegative components $s_{\kappa}, \kappa \in \mathcal{T}$, defined by

$$
H^{\mathrm{s}}(\Omega, \mathcal{T})=\left\{u \in L_{2}(\Omega):\left.u\right|_{\kappa} \in H^{s_{\kappa}}(\kappa) \quad \forall \kappa \in \mathcal{T}\right\} .
$$

If $s_{\kappa}=s \geq 0$ for all $\kappa \in \mathcal{T}$, we shall simply write $H^{s}(\Omega, \mathcal{T})$.

In the next section, we formulate the $h p$-version of the Discontinuous Galerkin Finite Element Method ( $h p$-DGFEM, for short) for the numerical solution of (2.1).

\subsection{The $h p$-discontinuous Galerkin method}

Given that $\kappa$ is an element in the subdivision $\mathcal{T}$, we denote by $\partial \kappa$ the union of $(d-1)$-dimensional open faces of $\kappa$. This is non-standard notation in that $\partial \kappa$ is a 
subset of the boundary of $\kappa$. Let $x \in \partial \kappa$ and suppose that $\mathbf{n}_{\kappa}(x)$ denotes the unit outward normal vector to $\partial \kappa$ at $x$. With these conventions, we define the inflow and outflow parts of $\partial \kappa$, respectively, by

$$
\begin{aligned}
& \partial_{-} \kappa=\left\{x \in \partial \kappa: \mathbf{b}(x) \cdot \mathbf{n}_{\kappa}(x)<0\right\}, \\
& \partial_{+} \kappa=\left\{x \in \partial \kappa: \mathbf{b}(x) \cdot \mathbf{n}_{\kappa}(x) \geq 0\right\} .
\end{aligned}
$$

For each $\kappa \in \mathcal{T}$ and any $v \in H^{1}(\kappa)$ we denote by $v_{\kappa}^{+}$the interior trace of $v$ on $\partial \kappa$ (the trace taken from within $\kappa$ ). Now consider an element $\kappa$ such that the set $\partial_{-} \kappa \backslash \Gamma_{-}$is nonempty; then for each $x \in \partial_{-} \kappa \backslash \Gamma_{-}$(with the exception of a set of $(d-1)$-dimensional measure zero) there exists a unique element $\kappa^{\prime}$, depending on the choice of $x$, such that $x \in \partial_{+} \kappa^{\prime}$. Suppose that $v \in H^{1}(\Omega, \mathcal{T})$. If $\partial_{-} \kappa \backslash \Gamma_{-}$is nonempty for some $\kappa \in \mathcal{T}$, then we define the outer trace $v_{\kappa}^{-}$of $v$ on $\partial_{-} \kappa \backslash \Gamma_{-}$relative to $\kappa$ as the inner trace $v_{\kappa^{\prime}}^{+}$relative to those elements $\kappa^{\prime}$ for which $\partial_{+} \kappa^{\prime}$ has intersection with $\partial_{-} \kappa \backslash \Gamma_{-}$of positive $(d-1)$-dimensional measure. We also introduce the jump of $v$ across $\partial_{-} \kappa \backslash \Gamma_{-}$:

$$
[v]_{\kappa}=v_{\kappa}^{+}-v_{\kappa}^{-} .
$$

Since below it will always be clear from the context which element $\kappa$ in the subdivision $\mathcal{T}$ the quantities $\mathbf{n}_{\kappa}, v_{\kappa}^{+}, v_{\kappa}^{-}$and $[v]_{\kappa}$ correspond to, for the sake of notational simplicity we shall suppress the letter $\kappa$ in the subscript and write, respectively, $\mathbf{n}$, $v^{+}, v^{-}$and $[v]$ instead.

For $v, w \in H^{1}(\Omega, \mathcal{T})$, we define the bilinear form

$$
\begin{aligned}
B_{\mathrm{DG}}(w, v)= & \sum_{\kappa \in \mathcal{T}} \int_{\kappa} \mathcal{L} w v \mathrm{~d} x-\sum_{\kappa \in \mathcal{T}} \int_{\partial_{-} \varsigma \Gamma_{-}}(\mathbf{b} \cdot \mathbf{n})[w] v^{+} \mathrm{d} s \\
& -\sum_{\kappa \in \mathcal{T}} \int_{\partial_{-} \kappa \cap \Gamma_{-}}(\mathbf{b} \cdot \mathbf{n}) w^{+} v^{+} \mathrm{d} s
\end{aligned}
$$

and, for $v \in H^{1}(\Omega, \mathcal{T})$, we consider the linear functional

$$
\ell_{\mathrm{DG}}(v)=\sum_{\kappa \in \mathcal{T}} \int_{\kappa} f v \mathrm{~d} x-\sum_{\kappa \in \mathcal{T}} \int_{\partial_{-} \kappa \cap \Gamma_{-}}(\mathbf{b} \cdot \mathbf{n}) g v^{+} \mathrm{d} s .
$$

The $h p$-DGFEM for (2.1) is defined as follows: find $u_{\mathrm{DG}} \in S^{\mathbf{p}}(\Omega, \mathcal{T}, \mathbf{F})$ such that

$$
B_{\mathrm{DG}}\left(u_{\mathrm{DG}}, v\right)=\ell_{\mathrm{DG}}(v) \quad \forall v \in S^{\mathbf{p}}(\Omega, \mathcal{T}, \mathbf{F}) .
$$

We note that

$$
\begin{aligned}
B_{\mathrm{DG}}(v, v)= & \sum_{\kappa} \int_{\kappa} c_{0}^{2}(x)|v|^{2} \mathrm{~d} x+\frac{1}{2} \sum_{\kappa} \int_{\partial_{-} \kappa \cap \Gamma_{-}}|\mathbf{b} \cdot \mathbf{n}|\left|v^{+}\right|^{2} \mathrm{~d} s \\
& +\frac{1}{2} \sum_{\kappa} \int_{\partial_{-} \kappa \backslash \Gamma_{-}}|\mathbf{b} \cdot \mathbf{n}||[v]|^{2} \mathrm{~d} s+\frac{1}{2} \sum_{\kappa} \int_{\partial_{+} \kappa \cap \Gamma_{+}}|\mathbf{b} \cdot \mathbf{n}|\left|v^{+}\right|^{2} \mathrm{~d} s
\end{aligned}
$$


for all $v \in H^{1}(\Omega, \mathcal{T})$ (see, for example, (2.31) in [14]), and therefore $B_{\mathrm{DG}}(v, v)>0$ for all $v \in H^{1}(\Omega, \mathcal{T}) \backslash\{0\}$; thus, recalling that (2.5) is a linear problem on the finitedimensional linear space $S^{\mathbf{p}}(\Omega, \mathcal{T}, \mathbf{F})$, we deduce the existence of a unique solution $u_{\mathrm{DG}}$ in $S^{\mathbf{p}}(\Omega, \mathcal{T}, \mathbf{F})$ to $(2.5)$. Further, for each $v \in H^{1}(\Omega, \mathcal{T})$,

$$
\begin{aligned}
\left|\ell_{\mathrm{DG}}(v)\right| \leq & \left(\sum_{\kappa \in \mathcal{T}} \int_{\kappa} c_{0}^{-2}(x)|f|^{2} \mathrm{~d} x+2 \sum_{\kappa \in \mathcal{T}} \int_{\partial_{-} \kappa \cap \Gamma_{-}}|\mathbf{b} \cdot \mathbf{n}||g|^{2} \mathrm{~d} s\right)^{1 / 2} \\
& \times\left(\sum_{\kappa \in \mathcal{T}} \int_{\kappa} c_{0}^{2}(x)|v|^{2} \mathrm{~d} x+\frac{1}{2} \sum_{\kappa \in \mathcal{T}} \int_{\partial_{-} \kappa \cap \Gamma_{-}}|\mathbf{b} \cdot \mathbf{n}|\left|v^{+}\right|^{2} \mathrm{~d} s\right)^{1 / 2} .
\end{aligned}
$$

Hence, letting $\|\mid v\|_{\mathrm{DG}}=\left[B_{\mathrm{DG}}(v, v)\right]^{1 / 2}$, we deduce the stability of the method in the sense that

$$
\left\|\left|u_{\mathrm{DG}}\right|\right\|_{\mathrm{DG}} \leq\left(\sum_{\kappa \in \mathcal{T}} \int_{\kappa} c_{0}^{-2}(x)|f(x)|^{2} \mathrm{~d} x+2 \sum_{\kappa \in \mathcal{T}} \int_{\partial_{-} \kappa \cap \Gamma_{-}}|\mathbf{b}(x) \cdot \mathbf{n}(x)||g(s)|^{2} \mathrm{~d} s\right)^{1 / 2} .
$$

For the a priori error analysis of (2.5) in the DG-norm $\||\cdot|\|_{\text {DG }}$ we refer to the paper [13], cf. Lemma 9 below. The analysis of the scheme (2.5) with the addition of streamline-diffusion stabilisation has been considered in the paper [14]; see also Bey \& Oden [6]. Here we shall concentrate on the situation when there is no streamlinediffusion stabilisation in the method.

\section{A posteriori and a priori error analysis}

In many problems of physical importance the quantity of interest is a linear functional $J(\cdot)$ of the solution. Relevant examples include the lift and drag coefficients for a body immersed into an inviscid fluid, the local mean value of the field, or its flux through the outflow boundary of the computational domain.

Suppose that we wish to control the discretisation error in some linear functional $J(\cdot)$ defined on a linear space which contains $H(\mathcal{L}, \Omega)+S^{\mathbf{p}}(\Omega, \mathcal{T}, \mathbf{F})$. Following the argument presented in [12] for stabilised continuous finite element approximations, we do so by deriving an a posteriori bound on the error between $J(u)$ and $J\left(u_{\mathrm{DG}}\right)$. We begin our analysis by considering the following dual or adjoint problem: find $z$ in $H\left(\mathcal{L}^{*}, \Omega\right)$ such that

$$
B_{\mathrm{DG}}(w, z)=J(w) \quad \forall w \in H(\mathcal{L}, \Omega)
$$

where $H\left(\mathcal{L}^{*}, \Omega\right)$ denotes the graph space of the adjoint operator $\mathcal{L}^{*}$ in $L_{2}(\Omega)$. Let us assume that (3.1) possesses a unique solution. Clearly, the validity of this assumption depends on the choice of the linear functional under consideration. Below, we present some important examples which are covered by our hypotheses. 
Example 1: Mean flow. Consider approximating the (weighted) mean value $J(\cdot) \equiv$ $M_{\psi}(\cdot)$ defined by

$$
J(w) \equiv M_{\psi}(w)=\int_{\Omega} w \psi \mathrm{d} x
$$

where $\psi \in L_{2}(\Omega)$ is a given weight function. In this case the dual problem takes the following form: find $z$ in $H\left(\mathcal{L}^{*}, \Omega\right)$ such that

$$
\begin{aligned}
\mathcal{L}^{*} z \equiv-\nabla \cdot(\mathbf{b} z)+c z & =\psi, \quad x \in \Omega \\
z & =0, \quad x \in \Gamma_{+} .
\end{aligned}
$$

Example 2: Point value. Under the assumption that the analytical solution $u$ is a continuous function in the neighbourhood of a given point $x_{c}$ in $\Omega$, the point value $u\left(x_{c}\right)$ may also be approximated. However, now $J(w)=w\left(x_{c}\right)$, and when this is inserted as right-hand side into the dual problem (3.1), the resulting weak solution $z$ is a measure rather than a regular distribution; in particular, $z$ does not belong to $L_{2}(\Omega)$. Thus, to avoid technical complications, we mollify the functional $J$ by considering a nonnegative function $\varphi$ in $L_{1, \text { loc }}\left(\mathbb{R}^{d}\right)$ whose support is contained in the unit ball $B(0,1)$ centred at $x=0$ and such that the integral of $\varphi$ over $B(0,1)$ is equal to 1 . Writing $\psi(x)=\varphi_{\varepsilon}(x) \equiv \varepsilon^{-d} \varphi\left(\left(x-x_{c}\right) / \varepsilon\right), M_{\psi}(u)$ converges to $u\left(x_{c}\right)$ as $\varepsilon \rightarrow 0$. Further, setting $J(w)=M_{\psi}(w)$ into (3.1) as right-hand side, for $0 \ll \varepsilon<1$ fixed, now results in a unique solution $z$ in $H\left(\mathcal{L}^{*}, \Omega\right)$.

Example 3: Outflow normal flux. As a final example, consider the (weighted) normal flux $J(\cdot) \equiv N_{\psi}(\cdot)$ through the outflow boundary $\Gamma_{+}$, defined, for $w \in H(\mathcal{L}, \Omega)$, by

$$
J(w) \equiv N_{\psi}(w)=\int_{\Gamma_{+}}(\mathbf{b} \cdot \mathbf{n}) w \psi \mathrm{d} s,
$$

where $\psi$ is a given 'weight' function in $L_{2}\left(\Gamma_{+}\right)$. A simple calculation based on the divergence theorem shows that $z$ is the (unique) solution to the following boundary value problem: find $z$ in $H\left(\mathcal{L}^{*}, \Omega\right)$ such that

$$
\begin{aligned}
\mathcal{L}^{*} z \equiv-\nabla \cdot(\mathbf{b} z)+c z & =0, \quad x \in \Omega, \\
z & =\psi, \quad x \in \Gamma_{+} .
\end{aligned}
$$

For a given linear functional $J(\cdot)$ the proceeding a posteriori error bound will be expressed in terms of the finite element residual $r_{h, p}$ defined on $\kappa \in \mathcal{T}$ by

$$
\left.r_{h, p}\right|_{\kappa}=\left.\left(f-\mathcal{L} u_{\mathrm{DG}}\right)\right|_{\kappa},
$$

which measures the extent to which $u_{\mathrm{DG}}$ fails to satisfy the differential equation on the union of the elements $\kappa$ in the mesh $\mathcal{T}$; thus we refer to $r_{h, p}$ as the internal residual. Also, since $u_{\mathrm{DG}}$ only satisfies the boundary conditions approximately, the 
difference $g-u_{\mathrm{DG}}$ is not necessarily zero on $\Gamma_{-}$; thus for each element $\kappa$ with $\partial_{-} \kappa \cap \Gamma_{-}$ of positive $(d-1)$-dimensional measure, we define the boundary residual $r_{h, p-}$ by

$$
\left.r_{h, p-}\right|_{\partial_{-} \kappa \cap \Gamma_{-}}=\left.\left(g-u_{\mathrm{DG}}^{+}\right)\right|_{\partial_{-} \kappa \cap \Gamma_{-}} .
$$

With these definitions we observe from (2.5) the following relationship between the internal and boundary residual:

$$
\begin{aligned}
B_{\mathrm{DG}}\left(u-u_{\mathrm{DG}}, v\right) \equiv & \sum_{\kappa \in \mathcal{T}}\left(r_{h, p}, v\right)_{\kappa}+\sum_{\kappa \in \mathcal{T}}\left((\mathbf{b} \cdot \mathbf{n})\left[u_{\mathrm{DG}}\right], v^{+}\right)_{\partial_{-}} \backslash \Gamma_{-} \\
& -\sum_{\kappa \in \mathcal{T}}\left((\mathbf{b} \cdot \mathbf{n}) r_{h, p-}, v^{+}\right)_{\partial_{-} \kappa \cap \Gamma_{-}}=0
\end{aligned}
$$

for all $v$ in $S^{\mathbf{p}}(\Omega, \mathcal{T}, \mathbf{F})$. The identity (3.5) is referred to as the Galerkin orthogonality property of the $h p$-DGFEM. We remark that the second term on the right-hand side of (3.5) reflects the fact that while the normal flux $(\mathbf{b} u) \cdot \mathbf{n}=(\mathbf{b} \cdot \mathbf{n}) u$ of the analytical solution $u$ is continuous across element interfaces (even if the analytical solution $u$ is only piecewise continuous on $\Omega$ ), the normal flux of the numerical solution $u_{\mathrm{DG}}$ is not, due to the choice of the finite element space $S^{\mathbf{p}}(\Omega, \mathcal{T}, \mathbf{F})$; for finite element approximations to (2.1) based on continuous piecewise polynomials, this term is, of course, equal to zero.

\subsection{Type I a posteriori error bound}

The starting point of the a posteriori error analysis is the following general result.

Theorem 1 Let $u$ and $u_{\mathrm{DG}}$ denote the solutions of (2.1) and (2.5), respectively, and suppose that the dual solution $z$ is defined by (3.1). Then, the following error representation formula holds:

$$
\begin{aligned}
J(u)-J\left(u_{\mathrm{DG}}\right)= & \sum_{\kappa \in \mathcal{T}}\left(r_{h, p}, z-z_{h, p}\right)_{\kappa}+\sum_{\kappa \in \mathcal{T}}\left((\mathbf{b} \cdot \mathbf{n})\left[u_{\mathrm{DG}}\right],\left(z-z_{h, p}\right)^{+}\right)_{\partial_{-} \kappa \backslash \Gamma_{-}} \\
& -\sum_{\kappa \in \mathcal{T}}\left((\mathbf{b} \cdot \mathbf{n}) r_{h, p-},\left(z-z_{h, p}\right)^{+}\right)_{\partial_{-} \kappa \cap \Gamma_{-}} \\
\equiv & \mathcal{E}_{\Omega}\left(u_{\mathrm{DG}}, h, p, z-z_{h, p}\right)
\end{aligned}
$$

for all $z_{h, p}$ in $S^{\mathbf{p}}(\Omega, \mathcal{T}, \mathbf{F})$.

Proof On choosing $w=u-u_{\mathrm{DG}}$ in (3.1) and recalling the linearity of $J(\cdot)$ and the Galerkin orthogonality property (3.5), we deduce that

$$
\begin{aligned}
J(u)-J\left(u_{\mathrm{DG}}\right)= & J\left(u-u_{\mathrm{DG}}\right)=B_{\mathrm{DG}}\left(u-u_{\mathrm{DG}}, z\right)=B_{\mathrm{DG}}\left(u-u_{\mathrm{DG}}, z-z_{h, p}\right) \\
= & \sum_{\kappa \in \mathcal{T}}\left(r_{h, p}, z-z_{h, p}\right)_{\kappa}+\sum_{\kappa \in \mathcal{T}}\left((\mathbf{b} \cdot \mathbf{n})\left[u_{\mathrm{DG}}\right],\left(z-z_{h, p}\right)^{+}\right)_{\partial_{-} \kappa \backslash \Gamma_{-}} \\
& -\sum_{\kappa \in \mathcal{T}}\left((\mathbf{b} \cdot \mathbf{n}) r_{h, p-},\left(z-z_{h, p}\right)^{+}\right)_{\partial_{-} \kappa \cap \Gamma_{-}},
\end{aligned}
$$


and hence (3.6).

Given a linear functional $J(\cdot)$ and a positive tolerance TOL, the aim of the computation is to calculate $u_{\mathrm{DG}}$ such that

$$
\left|J(u)-J\left(u_{\mathrm{DG}}\right)\right| \leq \mathrm{TOL} .
$$

According to (3.6), a necessary and sufficient condition for this to hold is that the stopping criterion

$$
\left|\mathcal{E}_{\Omega}\left(u_{\mathrm{DG}}, h, p, z-z_{h, p}\right)\right| \leq \mathrm{TOL}
$$

is satisfied. If (3.8) holds, then $J\left(u_{\mathrm{DG}}\right)$ is accepted as an accurate representation of $J(u)$; otherwise $u_{\mathrm{DG}}$ is discarded and a new, improved, approximation is computed on a refined subdivision. In order to ensure that the subdivision is refined only where necessary, a decision has to be made on each element $\kappa$ as to whether the local mesh-size $h_{\kappa}$ and the local polynomial degree $p_{\kappa}$ are acceptable in relation to TOL. A convenient approach to obtaining a local refinement criterion which relates the local discretisation parameters $h_{\kappa}$ and $p_{\kappa}$ to TOL is to localise $\mathcal{E}_{\Omega}\left(u_{\mathrm{DG}}, h, p, z-z_{h, p}\right)$. More precisely, $\left|\mathcal{E}_{\Omega}\left(u_{\mathrm{DG}}, h, p, z-z_{h, p}\right)\right|$ is further bounded above by noting that $\mathcal{E}_{\Omega}\left(u_{\mathrm{DG}}, h, p, z-z_{h, p}\right)$ can be decomposed as $\sum_{\kappa} \chi_{\kappa}$, with

$\chi_{\kappa}=\left(r_{h, p}, z-z_{h, p}\right)_{\kappa}+\left((\mathbf{b} \cdot \mathbf{n})\left[u_{\mathrm{DG}}\right],\left(z-z_{h, p}\right)^{+}\right)_{\partial_{-}} \backslash \Gamma_{-}+\left((\mathbf{b} \cdot \mathbf{n}) r_{h, p-},\left(z-z_{h, p}\right)^{+}\right)_{\partial_{-} \kappa \cap \Gamma_{-}}$,

and applying the inequality $\left|\sum_{\kappa} \chi_{\kappa}\right| \leq \sum_{\kappa}\left|\chi_{\kappa}\right|$. Thus we arrive at the following result.

Corollary 2 Under the assumptions of Theorem 1, the following a posteriori error bound holds:

$$
\left|J(u)-J\left(u_{\mathrm{DG}}\right)\right| \leq \mathcal{E}\left(u_{\mathrm{DG}}, h, p, z-z_{h, p}\right)
$$

where

$$
\mathcal{E}\left(u_{\mathrm{DG}}, h, p, z-z_{h, p}\right) \equiv \sum_{\kappa \in \mathcal{T}} \eta_{\kappa}
$$

and

$$
\begin{aligned}
\eta_{\kappa}= & \mid\left(r_{h, p}, z-z_{h, p}\right)_{\kappa}+\left((\mathbf{b} \cdot \mathbf{n})\left[u_{\mathrm{DG}}\right],\left(z-z_{h, p}\right)^{+}\right)_{\partial_{-} \kappa \backslash \Gamma_{-}} \\
& -\left((\mathbf{b} \cdot \mathbf{n}) r_{h, p-},\left(z-z_{h, p}\right)^{+}\right)_{\partial_{-} \kappa \cap \Gamma_{-}} \mid .
\end{aligned}
$$

Now, a possible local refinement criterion might, for example, consist of checking whether, on each element $\kappa$ in the subdivision $\mathcal{T}$, the inequality

$$
\eta_{\kappa} \leq \frac{\mathrm{TOL}}{N}
$$


holds, where $N$ is the number of elements in $\mathcal{T}$. If (3.12) is valid on each element $\kappa$ in $\mathcal{T}$ then, according to (3.9), the stopping criterion (3.8) has been reached and the required error control (3.7) has been achieved. The a posteriori error bound (3.9) - (3.11) where the difference between the dual solution $z$ and its discrete representation (e.g. interpolant, quasi-interpolant or projection) $z_{h, p}$ defined on the primal subdivision $\mathcal{T}$ enters into the error estimate as local weight function, will be referred to as an a posteriori error bound of Type I.

It may seem natural to further bound $\eta_{\kappa}$ in (3.11) by writing

$$
\begin{array}{r}
\eta_{\kappa} \leq\left|\left(r_{h, p}, z-z_{h, p}\right)_{\kappa}\right|+\left|\left((\mathbf{b} \cdot \mathbf{n})\left[u_{\mathrm{DG}}\right],\left(z-z_{h, p}\right)^{+}\right)_{\partial_{-} \kappa \backslash \Gamma_{-}}\right| \\
+\left|\left((\mathbf{b} \cdot \mathbf{n}) r_{h, p-},\left(z-z_{h, p}\right)^{+}\right)_{\partial_{-} \kappa \cap \Gamma_{-}}\right| \equiv \zeta_{\kappa} .
\end{array}
$$

We may then consider the a posteriori error bound

$$
\left|J(u)-J\left(u_{\mathrm{DG}}\right)\right| \leq \sum_{\kappa \in \mathcal{T}} \zeta_{\kappa} .
$$

Indeed, we could proceed even further, to eliminate $z_{h, p}$ from the a posteriori error estimate, by bounding each term in $\zeta_{\kappa}$ via the Cauchy-Schwarz inequality and standard results from approximation theory to bound the expressions $\left\|z-z_{h, p}\right\|_{L_{2}(\kappa)}$ and $\left\|\left(z-z_{h, p}\right)^{+}\right\|_{L_{2}\left(\partial_{-} \kappa\right)}$ in terms of the discretisation parameters $h_{\kappa}$ and $p_{\kappa}$ and Sobolev seminorms of $z$. For then, finally, the dual solution $z$ may also be eliminated from the a posteriori error estimate by bounding norms of $z$ by suitable norms of the data for the dual problem via hyperbolic well-posedness results. The resulting $a$ posteriori error bound will then, in the spirit of Johnson et al. [15], only involve the residual terms $r_{h p},(\mathbf{b} \cdot \mathbf{n})\left[u_{\mathrm{DG}}\right]$ and $r_{h, p-}$, the discretisation parameters, the interpolation constants and the stability factor of the dual problem; such an estimate will be referred to here as a Type II a posteriori error bound. We note that the residual-based weighted a posteriori error bounds of Becker and Rannacher [5] are intermediate between Type I and Type II bounds, although, due to the locality of the weight, closer in spirit to those of Type I. For earlier work on Type I and Type II error bounds for finite element and finite volume approximations to linear hyperbolic problems, we refer to [12] and [19].

The seemingly harmless transition from the Type I error bound (3.9) to (3.13) and the subsequent elimination of the dual solution $z$ en route to a Type II bound can be detrimental: due to loss of global cancellations the resulting Type II bound may, under mesh refinement, exhibit a rate of convergence inferior to that of $\mid J(u)-$ $J\left(u_{\mathrm{DG}}\right) \mid$, resulting in uneconomical meshes and an inefficient adaptive algorithm (see [12], for example). For this reason we refrain from bounding the terms $\eta_{\kappa}$ further; in particular, we shall not attempt to eliminate the dual solution $z$ from our $a$ posteriori error bound by exploiting standard approximation results and the strong stability of the dual problem. Instead, in the practical implementation of (3.9) we replace $z$ in the bound (3.9) by an approximation $\tilde{z}_{\mathrm{DG}}$, computed by an $h p$ adaptive discontinuous Galerkin finite element method on a sequence of auxiliary (dual) subdivisions $\tilde{\mathcal{T}}$ of the computational domain $\Omega$, see Section 4.1 below. We 
then define $z_{h, p}$ as the $L_{2}(\Omega)$ projection of $\tilde{z}_{\mathrm{DG}}$ onto the "primal" finite element space $S^{\mathbf{p}}(\Omega, \mathcal{T}, \mathbf{F})$ over the "primal" subdivision $\mathcal{T}$. Thus, after decomposing the error representation formula derived in Theorem 1 into terms which are computable, i.e. those involving the numerical approximation $\tilde{z}_{\mathrm{DG}}$ to the dual problem, and those that involve the analytical dual solution $z$, we arrive at the following result.

Corollary 3 Under the assumptions of Theorem 1, the following a posteriori error bound holds:

$$
\begin{aligned}
\left|J(u)-J\left(u_{\mathrm{DG}}\right)\right| & \leq \mathcal{E}\left(u_{\mathrm{DG}}, h, p, \tilde{z}_{\mathrm{DG}}-z_{h, p}\right)+\left|\mathcal{E}_{\Omega}\left(u_{\mathrm{DG}}, h, p, z-\tilde{z}_{\mathrm{DG}}\right)\right| \\
& \equiv \mathcal{E}_{\mathrm{P}}+\mathcal{E}_{\mathrm{D}},
\end{aligned}
$$

where $\mathcal{E}$ and $\mathcal{E}_{\Omega}$ are defined in (3.10) and (3.6), respectively.

We emphasise here that the fundamental difference between the terms $\mathcal{E}\left(u_{\mathrm{DG}}, h, p, \cdot\right)$ and $\left|\mathcal{E}_{\Omega}\left(u_{\mathrm{DG}}, h, p, \cdot\right)\right|$ is that in the former the absolute value signs appear under the summation over the elements $\kappa \in \mathcal{T}$, while in the latter the absolute value sign is outside the sum. It will be shown through numerical experiments that the true weighting function $z-z_{h, p}$ appearing in the a posteriori error bound (3.9) may be accurately approximated by $\tilde{z}_{\mathrm{DG}}-z_{h, p}$; indeed, we shall show that $\mathcal{E}_{\mathrm{P}}$ is typically an order of magnitude larger than $\mathcal{E}_{\mathrm{D}}$, cf. Table 1 and Figures 4 and 8 below, and [11] in the case of nonlinear hyperbolic conservation laws. Therefore, $\mathcal{E}_{\mathrm{D}}$ can be safely absorbed into $\mathcal{E}_{\mathrm{P}}$ without compromising the reliability of the adaptive algorithm when the stopping criterion (3.8) is replaced by

$$
\mathcal{E}_{\mathrm{P}} \leq \mathrm{TOL} .
$$

By this we mean that the right-hand side of (3.14) remains an upper bound on the true error in the linear functional $J(\cdot)$ even when the term $\mathcal{E}_{\mathrm{D}}$ is neglected in the process of error control for the primal problem.

The $h p$-DGFEM approximation $\tilde{z}_{\mathrm{DG}}$ to the dual solution $z$ appearing in $\mathcal{E}_{\mathrm{P}}$ will be computed by an $h p$-adaptive finite element algorithm, on a sequence of "dual meshes" $\tilde{\mathcal{T}}$ which, in general, differ from the "primal meshes" $\mathcal{T}$. The sequence of dual meshes will be generated by means of an a posteriori error bound on

$$
\mathcal{E}_{\mathrm{D}}=\left|\mathcal{E}_{\Omega}\left(u_{\mathrm{DG}}, h, p, z\right)-\mathcal{E}_{\Omega}\left(u_{\mathrm{DG}}, h, p, \tilde{z}_{\mathrm{DG}}\right)\right| .
$$

Since $\mathcal{E}_{\Omega}\left(u_{\mathrm{DG}}, h, p, \cdot\right)$ is a linear functional, we can derive an a posteriori bound on $\mathcal{E}_{\mathrm{D}}$ by mimicking the process of error estimation for the primal problem described above, with the primal problem replaced by the dual and the functional $J(\cdot)$ substituted by $\mathcal{E}_{\Omega}\left(u_{\mathrm{DG}}, h, p, \cdot\right)$. As the use of a Type I a posteriori error bound on $\mathcal{E}_{\mathrm{D}}$ would involve the solution of the dual to the dual problem which would then in turn have to be solved numerically, we shall instead use a crude Type II a posteriori error bound on $\mathcal{E}_{\mathrm{D}}$ so as to terminate the potentially infinite succession of mutually dual problems that would otherwise arise. The crudeness of the Type II bound on $\mathcal{E}_{\mathrm{D}}$ will be of no 
concern: as noted above, numerical experiments indicate that $\mathcal{E}_{\mathrm{D}} \ll \mathcal{E}_{\mathrm{P}}$, so from the practical point of view there appears to be little advantage in performing reliable error control for $\mathcal{E}_{\mathrm{D}}$; our aim, when using the Type II bound on $\mathcal{E}_{\mathrm{D}}$, is merely to generate an adequate sequence of finite element approximations $\tilde{z}_{\mathrm{DG}}$ to the dual solution $z$ which we can then use to compute $\mathcal{E}_{\mathrm{P}}$. The next section is, therefore, devoted to Type II a posteriori error bounds for the $h p$-DGFEM.

\subsection{Type II a posteriori error bounds}

For convenience, in the rest of this section we shall restrict ourselves to meshes consisting of affine equivalent $d$-parallelepiped elements. Generalisations to nonaffine elements may be treated by constructing meshes consisting of local patches as in [14].

Assuming that $m$ is a positive integer, we define the negative Sobolev norm $\|\cdot\|_{H^{-m}(\Omega)}$ in the usual way:

$$
\|w\|_{H^{-m}(\Omega)}=\sup _{v \in C_{0}^{\infty}(\Omega)} \frac{|(w, v)|}{\|v\|_{H^{m}(\Omega)}} .
$$

Further, we write $\|v\|_{\tau}, \tau \subset \partial \kappa, \kappa \in \mathcal{T}$, to denote the (semi)norm induced by the (semi)inner product

$$
\langle v, w\rangle_{\tau}=\int_{\tau}|\mathbf{b} \cdot \mathbf{n}| v w \mathrm{~d} s
$$

for $v, w \in L_{2}(\tau)$. Finally, we recall from [14] and [18] the following approximation results for the finite element space $S^{\mathbf{p}}(\Omega, \mathcal{T}, \mathbf{F})$.

Lemma 4 Suppose that $\left.u\right|_{\kappa} \in H^{k_{\kappa}}(\kappa), k_{\kappa} \geq 1$, for some $\kappa$ in $\mathcal{T}$. Then, there exists $\Pi_{h p} u$ in the finite element space $S^{\mathbf{p}}(\Omega, \mathcal{T}, \mathbf{F})$, a constant $C_{\text {int }}$ dependent only on $d$ and the shape-regularity of $\mathcal{T}$, but independent of $u, h_{\kappa}=\operatorname{diam}(\kappa), p_{\kappa}$ and $k_{\kappa}$, such that

$$
\left\|u-\Pi_{h p} u\right\|_{L_{2}(\kappa)}^{2} \leq C_{\mathrm{int}}^{2} h_{\kappa}^{2 s_{\kappa}} \frac{1}{p_{\kappa}\left(p_{\kappa}+1\right)} \Phi_{1}\left(p_{\kappa}, s_{\kappa}\right)|u|_{H^{s_{\kappa}(\kappa)}}^{2}
$$

and

$$
\left\|\nabla\left(u-\Pi_{h p} u\right)\right\|_{L_{2}(\kappa)}^{2} \leq C_{\mathrm{int}}^{2} h_{\kappa}^{2 s_{\kappa}-2} \Phi_{1}\left(p_{\kappa}, s_{\kappa}\right)|u|_{H^{s_{\kappa}(\kappa)}}^{2},
$$

for $1 \leq s_{\kappa} \leq \min \left(k_{\kappa}, p_{\kappa}+1\right)$. Here,

$$
\Phi_{1}(p, s):=\frac{\Gamma(p-s+2)}{\Gamma(p+s)}+\frac{1}{p(p+1)} \frac{\Gamma(p-s+3)}{\Gamma(p+s-1)}, \quad 1 \leq s \leq p+1 .
$$


Theorem 5 Let $u$ and $u_{\mathrm{DG}}$ denote the solutions of (2.1) and (2.5), respectively. Suppose further that the dual problem (3.1) has a unique solution $z \in H\left(\mathcal{L}^{*}, \Omega\right)$ such that $\left.z\right|_{\kappa} \in H^{t_{\kappa}}(\kappa)$ for each $\kappa \in \mathcal{T}$, with $1 \leq t_{\kappa} \leq p_{\kappa}+1$. Then,

$$
\begin{aligned}
\left|J(u)-J\left(u_{\mathrm{DG}}\right)\right| \leq C_{0} C_{\mathrm{int}}|z|_{H^{\mathrm{t}}(\Omega, \mathcal{T})} & {\left[\left(\sum_{\kappa \in \mathcal{T}} \frac{h_{\kappa}^{2 t_{\kappa}}}{p_{\kappa}\left(p_{\kappa}+1\right)} \Phi_{1}\left(p_{\kappa}, t_{\kappa}\right)\left\|r_{h, p}\right\|_{L_{2}(\kappa)}^{2}\right)^{1 / 2}\right.} \\
+ & \left(\sum_{\kappa \in \mathcal{T}} \frac{h_{\kappa}^{2 t_{\kappa}-1}}{p_{\kappa}} \Phi_{1}\left(p_{\kappa}, t_{\kappa}\right)\left\|\left[u_{\mathrm{DG}}\right]\right\|_{\partial_{-} \kappa \backslash \Gamma_{-}}^{2}\right)^{1 / 2} \\
+ & \left.\left(\sum_{\kappa \in \mathcal{T}} \frac{h_{\kappa}^{2 t_{\kappa}-1}}{p_{\kappa}} \Phi_{1}\left(p_{\kappa}, t_{\kappa}\right)\left\|r_{h, p-}\right\|_{\partial_{-} \kappa \cap \Gamma_{-}}^{2}\right)^{1 / 2}\right],
\end{aligned}
$$

where $C_{0}$ depends only on $\|\mathbf{b}\|_{L_{\infty}(\Omega)}$, the dimension $d$ and the shape-regularity of $\mathcal{T}$.

Proof Applying the Cauchy-Schwarz inequality to the right-hand side of (3.9) in Corollary 2 gives

$$
\begin{aligned}
\left|J(u)-J\left(u_{\mathrm{DG}}\right)\right| \leq & \sum_{\kappa \in \mathcal{T}}\left\|r_{h, p}\right\|_{L_{2}(\kappa)}\left\|z-z_{h, p}\right\|_{L_{2}(\kappa)} \\
& +\sum_{\kappa \in \mathcal{T}}\left\|\left[u_{\mathrm{DG}}\right]\right\|_{\partial_{-} \kappa \backslash \Gamma_{-}}\left\|\left(z-z_{h, p}\right)^{+}\right\|_{\partial_{-} \kappa \backslash \Gamma_{-}} \\
& +\sum_{\kappa \in \mathcal{T}}\left\|r_{h, p-}\right\|_{\partial_{-} \kappa \cap \Gamma_{-}}\left\|\left(z-z_{h, p}\right)^{+}\right\|_{\partial_{-} \kappa \cap \Gamma_{-}} \\
\equiv & \mathrm{I}+\mathrm{II}+\mathrm{III} .
\end{aligned}
$$

Exploiting the approximation result stated in Lemma 4, together with the Cauchy-Schwarz inequality, we get

$$
\mathrm{I} \leq C_{\text {int }}\left(\sum_{\kappa \in \mathcal{T}} \frac{h_{\kappa}^{2 t_{\kappa}}}{p_{\kappa}\left(p_{\kappa}+1\right)} \Phi_{1}\left(p_{\kappa}, t_{\kappa}\right)\left\|r_{h, p}\right\|_{L_{2}(\kappa)}^{2}\right)^{1 / 2}\left(\sum_{\kappa \in \mathcal{T}}|z|_{H^{t_{\kappa}(\kappa)}}^{2}\right)^{1 / 2}
$$

for $1 \leq t_{\kappa} \leq p_{\kappa}+1$ and $\kappa$ in $\mathcal{T}$. To bound the approximation error $z-z_{h, p}$ on a subset $\tau$ of the boundary of a given element $\kappa$ in $\mathcal{T}$, we use the following trace inequality:

$$
\|v\|_{\mathcal{\tau}}^{2} \leq \frac{1}{2} C_{0}^{2}\left(\|\nabla v\|_{L_{2}(\kappa)}\|v\|_{L_{2}(\kappa)}+h_{\kappa}^{-1}\|v\|_{L_{2}(\kappa)}^{2}\right) ;
$$

here, $C_{0}$ depends only on $\|\mathbf{b}\|_{L_{\infty}(\Omega)}, d$ and the shape-regularity of $\mathcal{T}$.

Exploiting (3.16) together with Lemma 4 gives the following bounds on terms II and III:

$$
\begin{aligned}
\text { II } & \leq C_{0} C_{\text {int }}\left(\sum_{\kappa \in \mathcal{T}} \frac{h_{\kappa}^{2 t_{\kappa}-1}}{p_{\kappa}} \Phi_{1}\left(p_{\kappa}, t_{\kappa}\right)\left\|\left[u_{\mathrm{DG}}\right]\right\|_{\partial_{-}}^{2} \backslash \Gamma_{-}\right)^{1 / 2}\left(\sum_{\kappa \in \mathcal{T}}|z|_{H^{t_{\kappa}}(\kappa)}^{2}\right)^{1 / 2}, \\
\mathrm{III} & \leq C_{0} C_{\text {int }}\left(\sum_{\kappa \in \mathcal{T}} \frac{h_{\kappa}^{2 t_{\kappa}-1}}{p_{\kappa}} \Phi_{1}\left(p_{\kappa}, t_{\kappa}\right)\left\|r_{h, p-}\right\|_{\partial_{-} \kappa \cap \Gamma_{-}}^{2}\right)^{1 / 2}\left(\sum_{\kappa \in \mathcal{T}}|z|_{H^{t_{\kappa}}(\kappa)}^{2}\right)^{1 / 2}
\end{aligned}
$$


for $1 \leq t_{\kappa} \leq p_{\kappa}+1$ and $\kappa$ in $\mathcal{T}$. Upon inserting the estimates on $I, I I$ and $I I I$ into (3.15) the result follows.

Remark 6 As an application of Theorem 5, let us consider the problem of a posteriori error estimation for the hp-DGFEM approximation of the weighted mean value of the solution $u$ to the model problem (2.1). Choosing $J(u)=M_{\psi}(u)$ in Theorem 5 with the weight function $\psi \in C_{0}^{\infty}(\Omega)$ gives

$$
\begin{aligned}
\left|M_{\psi}(u)-M_{\psi}\left(u_{\mathrm{DG}}\right)\right| \equiv & \left|\left(u-u_{\mathrm{DG}}, \psi\right)\right| \\
\leq & C_{0} C_{\mathrm{int}}\left[\left(\sum_{\kappa \in \mathcal{T}} \frac{h_{\kappa}^{2 t_{\kappa}}}{p_{\kappa}\left(p_{\kappa}+1\right)} \Phi_{1}\left(p_{\kappa}, t_{\kappa}\right)\left\|r_{h, p}\right\|_{L_{2}(\kappa)}^{2}\right)^{1 / 2}\right. \\
& +\left(\sum_{\kappa \in \mathcal{T}} \frac{h_{\kappa}^{2 t_{\kappa}-1}}{p_{\kappa}} \Phi_{1}\left(p_{\kappa}, t_{\kappa}\right)\left\|\left[u_{\mathrm{DG}}\right]\right\|_{\partial_{-} \varsigma \Gamma_{-}}^{2}\right)^{1 / 2} \\
& \left.+\left(\sum_{\kappa \in \mathcal{T}} \frac{h_{\kappa}^{2 t_{\kappa}-1}}{p_{\kappa}} \Phi_{1}\left(p_{\kappa}, t_{\kappa}\right)\left\|r_{h, p-}\right\|_{\partial_{-} \kappa \cap \Gamma_{-}}^{2}\right)^{1 / 2}\right]|z|_{H^{\mathbf{t}}(\Omega)} .
\end{aligned}
$$

Letting $m=\max _{\kappa \in \mathcal{T}} t_{\kappa}$, it follows by the Differentiability Theorem of Rauch [17] applied to (3.3) that

$$
\|z\|_{H^{m}(\Omega)} \leq C_{\text {stab }}\|\psi\|_{H^{m}(\Omega)}
$$

where $C_{\text {stab }}$ is a positive constant, independent of $\psi$, called the stability factor of the dual problem. Denoting by $\mathcal{B}\left(h, p, u_{\mathrm{DG}}\right)$ the expression in the square bracket in (3.18), we deduce the following Type II a posteriori error bound:

$$
\left|M_{\psi}(u)-M_{\psi}\left(u_{\mathrm{DG}}\right)\right| \leq C_{0} C_{\mathrm{int}} C_{\mathrm{stab}}\|\psi\|_{H^{m}(\Omega)} \mathcal{B}\left(h, p, u_{\mathrm{DG}}\right) .
$$

In fact, upon dividing both sides of the last inequality by $\|\psi\|_{H^{m}(\Omega)}$ and taking the supremum over all $\psi \in C_{0}^{\infty}(\Omega)$ ) yields the following Type II a posteriori error bound in a negative Sobolev norm:

$$
\left\|u-u_{\mathrm{DG}}\right\|_{H^{-m}(\Omega)} \leq C_{0} C_{\mathrm{int}} C_{\mathrm{stab}} \mathcal{B}\left(h, p, u_{\mathrm{DG}}\right) .
$$

We note that a bound identical to (3.18) holds for the weighted normal flux $N_{\psi}(u)$ of $u$ through the outflow boundary $\Gamma_{+}$, with $\psi$ a sufficiently smooth weight function defined on $\Gamma_{+}$.

\subsection{A priori error bounds}

As indicated in the Introduction, the $h p$-adaptive algorithms for the primal and dual problems will be driven by a posteriori error bounds: a Type I bound for the primal problem and a Type II bound for the dual problem. The decision about 
whether a local $h$ refinement/derefinement or a local $p$ refinement/derefinement is to be performed in the course of mesh adaptation will be based on assessing the local regularity of the primal and the dual solution. The estimation of local Sobolev indices which measure local regularity will, in turn, rely on an a priori bound on the error in the computed functional in terms of Sobolev norms of the analytical primal and dual solutions $u$ and $z$, respectively; this will indicate the expected rate of convergence for $\left|J(u)-J\left(u_{\mathrm{DG}}\right)\right|$ as the finite element space is enriched, i.e. as $h$ tends to 0 and $p$ tends to infinity, assuming that the primal and dual solutions have certain Sobolev regularities. The present section is devoted to the derivation of this a priori error bound.

We assume for the moment that the data for the problem (2.1) satisfy the following assumptions:

$$
\begin{array}{rr}
\mathbf{b} \cdot \nabla_{\mathcal{T}} v_{h} \in S^{\mathbf{p}}(\Omega, \mathcal{T}, \mathbf{F}) & \forall v_{h} \in S^{\mathbf{p}}(\Omega, \mathcal{T}, \mathbf{F}), \\
c \in S^{\mathbf{0}}(\Omega, \mathcal{T}, \mathbf{F}), \quad f \in S^{\mathbf{p}}(\Omega, \mathcal{T}, \mathbf{F}) .
\end{array}
$$

Here, $\nabla_{\mathcal{T}} v, v \in H^{1}(\Omega, \mathcal{T})$, denotes the broken gradient of $v$ defined by $\left.\left(\nabla_{\mathcal{T}} v\right)\right|_{\kappa}=$ $\nabla\left(\left.v\right|_{\kappa}\right), \kappa \in \mathcal{T}$. To ensure that (2.1) is then meaningful (i.e. that the characteristic curves of the differential operator $\mathcal{L}$ are correctly defined), we still assume that $\mathbf{b} \in\left[W_{\infty}^{1}(\Omega)\right]^{d}$.

Remark 7 The condition placed on $\mathbf{b}$ is required in the proof of the a priori error bound stated below (Lemma 9), cf. [13]; the conditions on the reaction/absorption term $c$ and the forcing function $f$ are required to ensure that the internal residual $r_{h, p}$ belongs to the finite element space $S^{\mathbf{p}}(\Omega, \mathcal{T}, \mathbf{F})$, cf. Lemma 10. However, we shall see in Section 5 that the restrictions (3.19) are not essential in practice for our a priori error bounds to hold.

Before embarking on the a priori error analysis, we quote from Schwab [18], Theorem 4.76, p. 208, the following inverse inequality for the finite element space $S^{\mathbf{p}}(\Omega, \mathcal{T}, \mathbf{F})$.

Lemma 8 Given $v$ in $S^{\mathbf{p}}(\Omega, \mathcal{T}, \mathbf{F})$, there exists a positive constant $C$, dependent only on $d$ and the shape-regularity of $\mathcal{T}$, such that

$$
|v|_{H^{1}(\kappa)} \leq C \frac{p_{\kappa}^{2}}{h_{\kappa}}\|v\|_{L_{2}(\kappa)}
$$

for all $\kappa$ in $\mathcal{T}$.

In the following lemma (cf. [13]) we formulate an a priori bound on the error $u-u_{\mathrm{DG}}$ in terms of the DG-norm $\|\cdot \mid\|_{\mathrm{DG}}$ defined in Section 2.2. We recall that

$$
\|v\| \|_{\mathrm{DG}}^{2}:=\sum_{\kappa \in \mathcal{T}}\left\{\left\|c_{0} v\right\|_{L_{2}(\kappa)}^{2}+\frac{1}{2}\left\|v^{+}\right\|_{\partial_{-} \kappa \cap \Gamma_{-}}+\frac{1}{2}\left\|v^{+}\right\|_{\partial_{+} \kappa \cap \Gamma_{+}}^{2}+\frac{1}{2}\left\|v^{+}-v^{-}\right\|_{\partial_{-} \kappa \backslash \Gamma_{-}}^{2}\right\},
$$

where $c_{0}$ is the (positive) function defined in (2.3). 
Lemma 9 Let $u$ and $u_{\mathrm{DG}}$ denote the solutions of (2.1) and (2.5), respectively. Assuming that (3.19a) holds and $\left.u\right|_{\kappa} \in H^{k_{\kappa}}(\kappa), k_{\kappa} \geq 1$, for all $\kappa$ in $\mathcal{T}$, we have

$$
\left|\left\|u-\left.u_{\mathrm{DG}}\left|\|_{\mathrm{DG}}^{2} \leq C \sum_{\kappa \in \mathcal{T}} h_{\kappa}^{2 s_{\kappa}-1} \Phi_{2}\left(p_{\kappa}, s_{\kappa}\right)\right| u\right|_{H^{s_{\kappa}}(\kappa)} ^{2},\right.\right.
$$

for any integers $1 \leq s_{\kappa} \leq \min \left(p_{\kappa}+1, k_{\kappa}\right), \kappa \in \mathcal{T}$. Here,

$$
\begin{aligned}
\Phi_{2}(s, p)= & \frac{1}{2 p+1}\left(\frac{\Gamma(p+2-s)}{\Gamma(p+s)}+\frac{\Gamma(p+3-s)}{\Gamma(p+1+s)}\right) \\
& +\left(\frac{\Gamma(p+2-s)}{\Gamma(p+2+s)}\right)^{\frac{1}{2}}\left(\frac{\Gamma(p+3-s)}{\Gamma(p+1+s)}\right)^{\frac{1}{2}}+\frac{\Gamma(p+2-s)}{\Gamma(p+2+s)}
\end{aligned}
$$

and $C$ is a positive constant depending only on $d,\|\mathbf{b}\|_{L_{\infty}(\Omega)},\|c\|_{L_{\infty}(\Omega)}$ and the shaperegularity of $\mathcal{T}$.

Furthermore, we need the following bound on the internal residual $r_{h, p}$.

Lemma 10 Assuming that the conditions on the data (3.19) hold, there exists a positive constant $C$, dependent only on $d$ and the shape-regularity of $\mathcal{T}$, such that

$$
\sum_{\kappa \in \mathcal{T}} \frac{h_{\kappa}}{p_{\kappa}^{2}+1}\left\|r_{h, p}\right\|_{L_{2}(\kappa)}^{2} \leq C \mid\left\|u-u_{\mathrm{DG}}\right\|_{\mathrm{DG}}^{2} .
$$

Proof From the Galerkin orthogonality property (3.5) we have

$$
\sum_{\kappa \in \mathcal{T}}\left(r_{h, p}, v\right)_{\kappa}=-\sum_{\kappa \in \mathcal{T}}\left((\mathbf{b} \cdot \mathbf{n})\left[u_{\mathrm{DG}}\right], v^{+}\right)_{\partial_{-}} \backslash \Gamma_{-}+\sum_{\kappa \in \mathcal{T}}\left((\mathbf{b} \cdot \mathbf{n}) r_{h, p-}, v^{+}\right)_{\partial_{-} \kappa \cap \Gamma_{-}}
$$

for any $v$ in $S^{\mathbf{p}}(\Omega, \mathcal{T}, \mathbf{F})$. Under the conditions (3.19), the internal residual $r_{h, p}$ belongs to the finite element space $S^{\mathbf{p}}(\Omega, \mathcal{T}, \mathbf{F})$; thereby choosing $v=\theta r_{h, p}$ in (3.21), where $\theta$ is in $S^{\mathbf{0}}(\Omega, \mathcal{T}, \mathbf{F})$ such that $\left.\theta\right|_{\kappa}=\theta_{\kappa}>0$, and applying the Cauchy-Schwarz inequality gives

$$
\sum_{\kappa \in \mathcal{T}} \theta_{\kappa}\left\|r_{h, p}\right\|_{L_{2}(\kappa)}^{2} \leq \sum_{\kappa \in \mathcal{T}} \theta_{\kappa}\left\|\left[u_{\mathrm{DG}}\right]\right\|_{\partial_{-} \backslash \Gamma_{-}}\left\|r_{h, p}^{+}\right\|\left\|_{\partial_{-} \kappa \backslash \Gamma_{-}}+\sum_{\kappa \in \mathcal{T}} \theta_{\kappa}\right\| r_{h, p-}\left\|_{\partial_{-} \kappa \cap \Gamma_{-}}\right\| r_{h, p}^{+} \|_{\partial_{-} \kappa \cap \Gamma_{-}} .
$$

Exploiting the trace inequality (3.16) together with the inverse inequality stated in Lemma 8 , we deduce that

$$
\sum_{\kappa \in \mathcal{T}} \theta_{\kappa}\left\|r_{h, p}\right\|_{L_{2}(\kappa)}^{2} \leq C \sum_{\kappa \in \mathcal{T}} \theta_{\kappa} \frac{p_{\kappa}^{2}+1}{h_{\kappa}}\left(\left\|\left[u_{\mathrm{DG}}\right]\right\|_{\partial_{-}}^{2} \backslash \backslash \Gamma_{-}+\frac{1}{2}\left\|r_{h, p-}\right\|_{\partial_{-} \kappa \cap \Gamma_{-}}^{2}\right) .
$$

Choosing $\theta_{\kappa}=h_{\kappa} /\left(p_{\kappa}^{2}+1\right)$ together with the definition of the DG-norm (3.20) gives the desired result.

Equipped with Lemmas 9 and 10, together with the approximation results stated in Lemma 4 , we are now in a position to prove the following $h p$-bound on the error in the computed functional $J(\cdot)$. 
Theorem 11 Let $u$ and $u_{\mathrm{DG}}$ denote the solutions of (2.1) and (2.5), respectively. Given that $\left.u\right|_{\kappa} \in H^{k_{\kappa}}(\kappa), k_{\kappa} \geq 1$, and $\left.z\right|_{\kappa} \in H^{l_{\kappa}}(\kappa), l_{\kappa} \geq 1$, for all $\kappa$ in $\mathcal{T}$, we have

$$
\begin{aligned}
\left|J(u)-J\left(u_{\mathrm{DG}}\right)\right|^{2} \leq C \sum_{\kappa \in \mathcal{T}} h_{\kappa}^{2 s_{\kappa}-1} \Phi_{2}\left(p_{\kappa}, s_{\kappa}\right)|u|_{H^{s_{\kappa}(\kappa)}}^{2} & \times \sum_{\kappa \in \mathcal{T}} h_{\kappa}^{2 t_{\kappa}-1} \Phi_{1}\left(p_{\kappa}, t_{\kappa}\right)|z|_{H^{t_{\kappa}(\kappa)}}^{2},
\end{aligned}
$$

for any $1 \leq s_{\kappa} \leq \min \left(p_{\kappa}+1, k_{\kappa}\right), 1 \leq t_{\kappa} \leq \min \left(p_{\kappa}+1, l_{\kappa}\right), \kappa \in \mathcal{T}$. Here, $C$ is a positive constant depending only on $d,\|\mathbf{b}\|_{L_{\infty}(\Omega)},\|c\|_{L_{\infty}(\Omega)}$ and the shape-regularity of $\mathcal{T}$.

Proof With terms I, II and III defined as in the proof of Theorem 5, cf. Equation (3.15), we deduce from Lemma 4 and (3.16), the following bounds

$$
\begin{aligned}
\mathrm{I} & \leq C\left(\sum_{\kappa \in \mathcal{T}} \frac{h_{\kappa}}{p_{\kappa}^{2}+1}\left\|r_{h, p}\right\|_{L_{2}(\kappa)}^{2}\right)^{1 / 2}\left(\sum_{\kappa \in \mathcal{T}} h_{\kappa}^{2 t_{\kappa}-1} \frac{p_{\kappa}^{2}+1}{p_{\kappa}\left(p_{\kappa}+1\right)} \Phi_{1}\left(p_{\kappa}, t_{\kappa}\right)|z|_{H^{t_{\kappa}(\kappa)}}^{2}\right)^{1 / 2},(3,2 \\
\mathrm{II} & \leq C\left(\sum_{\kappa \in \mathcal{T}}\left\|\left[u_{\mathrm{DG}}\right]\right\|_{\partial_{-} \kappa \backslash \Gamma_{-}}^{2}\right)^{1 / 2}\left(\sum_{\kappa \in \mathcal{T}} \frac{h_{\kappa}^{2 t_{\kappa}-1}}{p_{\kappa}} \Phi_{1}\left(p_{\kappa}, t_{\kappa}\right)|z|_{H^{t_{\kappa}(\kappa)}}^{2}\right)^{1 / 2}, \\
\mathrm{III} & \leq C\left(\sum_{\kappa \in \mathcal{T}}\left\|r_{h, p-}\right\|_{\partial_{-} \kappa \cap \Gamma_{-}}^{2}\right)^{1 / 2}\left(\sum_{\kappa \in \mathcal{T}} \frac{h_{\kappa}^{2 t_{\kappa}-1}}{p_{\kappa}} \Phi_{1}\left(p_{\kappa}, t_{\kappa}\right)|z|_{H^{t_{\kappa}}(\kappa)}^{2}\right)^{1 / 2}
\end{aligned}
$$

respectively. Collecting the bounds (3.23), (3.24) and (3.25), and exploiting Lemma 10 we get

$$
\left|J(u)-J\left(u_{\mathrm{DG}}\right)\right|^{2} \leq\left. C\left|\left\|u-u_{\mathrm{DG}}\right\|_{\mathrm{DG}}^{2} \sum_{\kappa \in \mathcal{T}} h_{\kappa}^{2 t_{\kappa}-1} \Phi_{1}\left(p_{\kappa}, t_{\kappa}\right)\right| z\right|_{H^{t_{\kappa}}(\kappa)} ^{2} .
$$

Finally, employing Lemma 9 gives the desired result.

Let us now discuss some special cases of the general error bound derived in Theorem 11. We first note that, for fixed $s$, Stirling's formula implies

$$
\Phi_{1}(p, s) \leq C(s) p^{-2 s+2}, \quad \Phi_{2}(p, s) \leq C(s) p^{-2 s+1},
$$

as $p \rightarrow \infty$. Thereby, for uniform orders $p_{\kappa}=p, s_{\kappa}=s, t_{\kappa}=t, k_{\kappa}=k, l_{\kappa}=l, s, t$, $k$ and $l$ integers, and $h_{\kappa}=h$ for all $\kappa$ in $\mathcal{T}$, we get the bound

$$
\left|J(u)-J\left(u_{\mathrm{DG}}\right)\right| \leq C\left(\frac{h}{p}\right)^{s+t-1} p^{1 / 2}|u|_{H^{s}(\Omega)}|z|_{H^{t}(\Omega)},
$$

where $1 \leq s \leq \min (p+1, k)$ and $1 \leq t \leq \min (p+1, l)$. Hence, we deduce that

$$
\left|J(u)-J\left(u_{\mathrm{DG}}\right)\right| \leq C \frac{h^{s+t-1}}{p^{k+l-1}} p^{1 / 2}\|u\|_{H^{k}(\Omega)}\|z\|_{H^{l}(\Omega)},
$$


where $1 \leq s \leq \min (p+1, k)$ and $1 \leq t \leq \min (p+1, l)$, cf. [20]; we note that in the transition from (3.27) to (3.28) the generic constant $C$ is increased by the factor $(k-1)^{k-1}(l-1)^{l-1}$. Here, the bounds (3.27) and (3.28) are optimal in $h$ and suboptimal in $p$ by $p^{1 / 2}$; in the case of fixed $p,(3.27)$, (3.28) reduce to the optimal $h$-convergence error bound proved in [12] for a stabilised continuous approximation to $u$. From (3.28) we may deduce the following a priori error bound

$$
\left\|u-u_{\mathrm{DG}}\right\|_{H^{-m}(\Omega)} \leq C \frac{h^{s+\theta-1}}{p^{k+m-1}} p^{1 / 2}\|u\|_{H^{k}(\Omega)},
$$

where $1 \leq s \leq \min (p+1, k)$ and $1 \leq \theta \leq \min (p+1, m)$. In the presence of streamline-diffusion stabilisation, with stabilisation parameter $\delta=h / p$, the bounds (3.27), (3.28) and (3.29) can be sharpened to ones that are simultaneously optimal in both $h$ and $p$.

The explicit dependence of the error bound stated in Theorem 11 on the local regularity of the primal and dual solutions $u$ and $z$, respectively, allows us to deduce that the error in the functional $J(\cdot)$ is exponentially convergent as $p_{\kappa} \rightarrow \infty$ for each $\kappa$ in $\mathcal{T}$. To this end, let us assume that that $z$ is elementwise analytic in the sense that, for each $\kappa \in \mathcal{T},\left.z\right|_{\kappa}$ has analytic extension to an open set, independent of $h_{\kappa}$, containing $\bar{\kappa}$. Then,

$$
\forall \kappa \in \mathcal{T} \quad \exists d_{\kappa}>1 \quad \exists C(z)>0 \quad \forall s>0:|z|_{H^{s}(\kappa)} \leq C(z)\left(d_{\kappa}\right)^{s} s ![\operatorname{meas}(\kappa)]^{1 / 2} .
$$

In order to emphasise the dependence of $d_{\kappa}$ on the particular function $z$ under consideration, we write $d_{\kappa}(z)$ in lieu of $d_{\kappa}$. Thereby, assuming that $z$ is elementwise analytic and $h_{\kappa}>0$ is fixed for all $\kappa$ in $\mathcal{T}$, on setting $t_{\kappa}=\alpha_{\kappa} p_{\kappa}+1$, where $0<\alpha_{\kappa}=$ $\left(1+\left(d_{\kappa}(z)\right)^{2}\right)^{-1 / 2}<1$ for all $\kappa \in \mathcal{T}$, it can be shown that

$$
\sum_{\kappa \in \mathcal{T}} h_{\kappa}^{2 t_{\kappa}-1} \Phi_{1}\left(p_{\kappa}, t_{\kappa}\right)|z|_{H^{t_{\kappa}(\kappa)}}^{2} \leq C(z) \sum_{\kappa \in \mathcal{T}} h_{\kappa}^{2 t_{\kappa}-1} p_{\kappa}^{3} \mathrm{e}^{-2 \lambda_{\kappa} p_{\kappa}} \operatorname{meas}(\kappa),
$$

where $\lambda_{\kappa}$ is a positive constant on each element $\kappa$ in the mesh $\mathcal{T}$; namely,

$$
\lambda_{\kappa}=\frac{1}{2}\left|\log F\left(\alpha_{\kappa}, d_{\kappa}(z)\right)\right|, \quad \text { where } \quad F(\alpha, d)=\frac{(1-\alpha)^{1-\alpha}}{(1+\alpha)^{1+\alpha}}(\alpha d)^{2 \alpha},
$$

see [14] for details. Similarly, assuming that $u$ is elementwise analytic, setting $s_{\kappa}=$ $\beta_{\kappa} p_{\kappa}+1$, where $0<\beta_{\kappa}=\left(1+\left(d_{\kappa}(u)\right)^{2}\right)^{-1 / 2}<1$ for all $\kappa \in \mathcal{T}$, we have that

$$
\sum_{\kappa \in \mathcal{T}} h_{\kappa}^{2 s_{\kappa}-1} \Phi_{2}\left(p_{\kappa}, s_{\kappa}\right)|u|_{H^{s_{\kappa}(\kappa)}}^{2} \leq C(u) \sum_{\kappa \in \mathcal{T}} h_{\kappa}^{2 s_{\kappa}-1} p_{\kappa}^{2} \mathrm{e}^{-2 \mu_{\kappa} p_{\kappa}} \operatorname{meas}(\kappa),
$$

where $\mu_{\kappa}=(1 / 2)\left|\log F\left(\beta_{\kappa}, d_{\kappa}(u)\right)\right|$. Thereby, combining (3.30) and (3.31), we deduce the exponential convergence estimate

$$
\left|J(u)-J\left(u_{\mathrm{DG}}\right)\right|^{2} \leq K \sum_{\kappa \in \mathcal{T}} h_{\kappa}^{2 s_{\kappa}-1} p_{\kappa}^{2} \mathrm{e}^{-2 \mu_{\kappa} p_{\kappa}} \operatorname{meas}(\kappa) \sum_{\kappa \in \mathcal{T}} h_{\kappa}^{2 t_{\kappa}-1} p_{\kappa}^{3} \mathrm{e}^{-2 \lambda_{\kappa} p_{\kappa}} \operatorname{meas}(\kappa)(3
$$

where $K=C(u) C(z)$. 
Remark 12 The bound (3.32) indicates that in order to ensure that the error in the functional $J(\cdot)$ decays exponentially as the degree of the approximating polynomial is increased, it is only necessary to assume that either $u$ or $z$ is elementwise analytic; this will be demonstrated numerically in Section 5.

\section{Implementational issues}

\subsection{Numerical approximation of the dual solution}

In this section we formulate the discontinuous Galerkin finite element approximation of the dual problem (3.1). As stated in Section 3, the particular form of the dual problem is dependent on the functional under consideration. For generality, let us suppose that $z$ is the (unique) solution to the following problem: find $z \in H\left(\mathcal{L}^{*}, \Omega\right)$ such that

$$
\begin{aligned}
\mathcal{L}^{*} z \equiv-\nabla \cdot(\mathbf{b} z)+c z & =\varphi, \quad x \in \Omega, \\
z & =\chi, \quad x \in \Gamma_{+} .
\end{aligned}
$$

Clearly, (4.1) covers both the case when the functional $J(\cdot)$ under consideration represents the local mean value of the solution $u$ and when $J(\cdot)$ is the outflow normal flux of $u$; cf. Section 3.

As in Section 2.1, we define $\tilde{S}^{\tilde{\mathbf{p}}}(\Omega, \tilde{\mathcal{T}}, \tilde{\mathbf{F}})$ to be the finite element space consisting of piecewise polynomials of degree $\left.\tilde{\mathbf{p}}\right|_{\tilde{\kappa}}=\tilde{p}_{\tilde{\kappa}}$ on a mesh $\tilde{\mathcal{T}}$ consisting of shape-regular elements $\tilde{\kappa}$ of size $\tilde{h}_{\tilde{\kappa}}$. With $\partial_{+} \tilde{\kappa}$ defined as in (2.4), we introduce the bilinear form

$$
\begin{aligned}
\tilde{B}_{\mathrm{DG}}(w, v)= & \sum_{\tilde{\kappa} \in \tilde{\mathcal{T}}} \int_{\tilde{\kappa}} \mathcal{L}^{*} w v \mathrm{~d} x+\sum_{\tilde{\kappa} \in \tilde{\mathcal{T}}} \int_{\partial_{+} \tilde{\kappa} \backslash \Gamma_{+}}(\mathbf{b} \cdot \mathbf{n})[w] v^{+} \mathrm{d} s \\
& +\sum_{\tilde{\kappa} \in \tilde{\mathcal{T}}} \int_{\partial_{+} \cap \tilde{\kappa} \cap \Gamma_{+}}(\mathbf{b} \cdot \mathbf{n}) w^{+} v^{+} \mathrm{d} s
\end{aligned}
$$

and linear functional

$$
\tilde{\ell}_{\mathrm{DG}}(v)=\sum_{\tilde{\kappa} \in \tilde{\mathcal{T}}} \int_{\tilde{\kappa}} \varphi v \mathrm{~d} x+\sum_{\tilde{\kappa} \in \tilde{\mathcal{T}}} \int_{\partial_{+} \tilde{\kappa} \cap \Gamma_{+}}(\mathbf{b} \cdot \mathbf{n}) \chi v^{+} \mathrm{d} s
$$

associated with the $h p$-DGFEM approximation of the dual problem (4.1). Now the $h p$-DGFEM for (4.1) is defined as follows: find $\tilde{z}_{\mathrm{DG}} \in \tilde{S} \tilde{\mathbf{p}}(\Omega, \tilde{\mathcal{T}}, \tilde{\mathbf{F}})$ such that

$$
\tilde{B}_{\mathrm{DG}}\left(\tilde{z}_{\mathrm{DG}}, v\right)=\tilde{\ell}_{\mathrm{DG}}(v) \quad \forall v \in \tilde{S}^{\tilde{\mathbf{p}}}(\Omega, \tilde{\mathcal{T}}, \tilde{\mathbf{F}}) .
$$

\subsection{Adaptive Algorithm}

For a user-defined tolerance TOL, we now consider the problem of designing the $h p$-finite element space $S^{\mathbf{p}}(\Omega, \mathcal{T}, \mathbf{F})$ such that

$$
\left|J(u)-J\left(u_{\mathrm{DG}}\right)\right| \leq \mathrm{TOL},
$$


subject to the constraint that the total number of degrees of freedom in $S^{\mathbf{p}}(\Omega, \mathcal{T}, \mathbf{F})$ is minimised. Following the discussion presented in Section 3, we exploit the $a$ posteriori error bound (3.14) to construct $S^{\mathbf{p}}(\Omega, \mathcal{T}, \mathbf{F})$ such that

$$
\mathcal{E}_{\mathrm{P}} \leq \mathrm{TOL}
$$

The stopping criterion (4.4) is enforced by equidistributing $\left.\mathcal{E}_{\mathrm{P}}\right|_{\kappa} \equiv \tilde{\eta}_{\kappa}$ over the elements $\kappa$ in the primal mesh $\mathcal{T}$, where $\tilde{\eta}_{\kappa}$ is defined in a similar manner to $\eta_{\kappa}$ with $z$ replaced by $\tilde{z}_{\mathrm{DG}}$ in (3.11). Thus, we insist that

$$
\tilde{\eta}_{\kappa} \approx \frac{\mathrm{TOL}}{N}
$$

holds for each $\kappa$ in $\mathcal{T}$; here, $N$ denotes the number of elements in the mesh $\mathcal{T}$.

Thereby, each of the elements in the primal mesh is flagged for either refinement or derefinement to ensure that the equidistribution principle (4.5) holds. Once an element $\kappa$ has been flagged a decision must be made whether the local mesh size $h_{\kappa}$ or the local degree $p_{\kappa}$ of the approximating polynomial should be adjusted accordingly. Let us first deal with refinement, i.e. when the local error estimator $\tilde{\eta}_{\kappa}$ is larger than the 'localised-tolerance' TOL/ $N$. Clearly, if the primal or dual solutions $u$ and $z$, respectively, are locally 'smooth', then $p$-enrichment will be more effective than $h$-refinement, since the error will be expected to decay quickly within the current element $\kappa$ as $p_{\kappa}$ is increased. However, if $u$ or $z$ have low regularity within the element $\kappa$, then $h$-refinement will be performed. Thus, regions in the computational domain where the primal or dual solution are locally non-smooth are isolated from smooth regions, thereby reducing the influence of singularities/discontinuities as well as making $p$-enrichment more effective.

To ensure that the desired level of accuracy is achieved efficiently, an automatic procedure for deciding when to $h$ - or $p$-refine must be implemented. To this end, we first compute the local error indicator $\tilde{\eta}_{\kappa}$ on each element $\kappa$ in the mesh $\mathcal{T}$ using both a $p_{\kappa}$ and a $p_{\kappa}-1$ representation for $u_{\mathrm{DG}}$; we denote the corresponding values of $\tilde{\eta}_{\kappa}$ by $\tilde{\eta}_{\kappa}\left(p_{\kappa}\right)$ and $\tilde{\eta}_{\kappa}\left(p_{\kappa}-1\right)$, respectively. Thereby, assuming that $\tilde{\eta}_{\kappa}\left(p_{\kappa}-1\right) \neq 0$, the perceived smoothness of the primal and dual solutions may be estimated using the ratio

$$
\rho_{\kappa}=\tilde{\eta}_{\kappa}\left(p_{\kappa}\right) / \tilde{\eta}_{\kappa}\left(p_{\kappa}-1\right),
$$

cf. Adjerid et al. [1] and Gui \& Babuška [10], for example. If $\rho_{\kappa} \leq \gamma, 0<\gamma<1$, the error is decreasing as the polynomial degree is increased, indicating that $p$ enrichment should be performed. On the other hand, $\rho_{\kappa}>\gamma$ means that the element $\kappa$ should be locally subdivided. The number $\gamma$ is referred to as the type-parameter [10]. Clearly, the choice of $\gamma$ is critical to the success of this algorithm and will depend on the asymptotic behaviour of the quantity of interest. Instead of assigning an $a d$ hoc value to the type parameter $\gamma$, we use $\rho_{\kappa}$ together with the a priori error bound (3.22) to directly estimate the local regularities $k_{\kappa}$ and $l_{\kappa}$ of the primal and 
dual solutions, respectively, on each element $\kappa$ in $\mathcal{T}$. More precisely, motivated by (3.28), we assume that on a given element $\kappa$ in $\mathcal{T}$

$$
\tilde{\eta}_{\kappa}=\left.\mathcal{E}_{\mathrm{P}}\right|_{\kappa} \approx C_{\kappa} p_{\kappa}^{-k_{\kappa}-l_{\kappa}+1}
$$

Thus, we have that

$$
k_{\kappa}+l_{\kappa}=\log \left(\rho_{\kappa}\right) / \log \left(\left(p_{\kappa}-1\right) / p_{\kappa}\right)+1 .
$$

Ideally, we would like to know $k_{\kappa}$ and $l_{\kappa}$ individually. To do so, we compute an estimate of $l_{\kappa}$. The dual regularity $l_{\kappa}$ may be estimated by calculating the $L^{2}(\kappa)$ norm of the error between the projection of $\tilde{z}_{\mathrm{DG}}$ in $\tilde{S}^{\tilde{\mathbf{p}}}(\Omega, \tilde{\mathcal{T}}, \tilde{\mathbf{F}})$ onto the finite element spaces $S^{\mathbf{p}}(\Omega, \mathcal{T}, \mathbf{F})$ and $S^{\mathbf{p}-\mathbf{1}}(\Omega, \mathcal{T}, \mathbf{F})$, together with the approximation results derived in [3]. Once both $k_{\kappa}$ and $l_{\kappa}$ have been determined on element $\kappa$, then $\kappa$ is $p$-enriched if either $k_{\kappa}$ or $l_{\kappa}$ is larger than $p_{\kappa}+1$; otherwise the element is subdivided. For computational simplicity, only one hanging node is allowed on each side of a given element $\kappa$; additionally, we restrict the variation in the polynomial degree vector $\mathbf{p}$ to be at most one between neighbouring elements. We note that this approach has been developed by Ainsworth \& Senior [2] in the context of norm control for second-order elliptic problems.

On the other hand, if an element has been flagged for derefinement, then the strategy implemented here is to coarsen the mesh in low-error-regions where either the primal or dual solutions $u$ and $z$, respectively, are smooth and decrease the degree of the approximating polynomial in low-error-regions when both $u$ or $z$ are not sufficiently regular, cf. [1]. To this end, we again compute the local regularities $k_{\kappa}$ and $l_{\kappa}$ of the primal and dual solutions, respectively, on each element $\kappa$ in $\mathcal{T}$ as described above. The element $\kappa$ is then coarsened if either $k_{\kappa}$ or $l_{\kappa}$ is larger than $p_{\kappa}+1$, otherwise the degree $p_{\kappa}$ is reduced by one.

The finite element space $\tilde{S} \tilde{\mathbf{p}}(\Omega, \tilde{\mathcal{T}}, \tilde{\mathbf{F}})$ and the finite element approximation $\tilde{z}_{\mathrm{DG}} \in$ $\tilde{S}^{\tilde{\mathbf{p}}}(\Omega, \tilde{\mathcal{T}}, \tilde{\mathbf{F}})$ of the dual solution $z$ will be constructed adaptively at the same time as $S^{\mathbf{p}}(\Omega, \mathcal{T}, \mathbf{F})$. To this end, we note that $\mathcal{E}_{\Omega}\left(u_{\mathrm{DG}}, h, p, \cdot\right)$ is a linear functional on $H\left(\mathcal{L}^{*}, \Omega\right)$, and we define the error indicator

$$
\begin{aligned}
\eta_{\tilde{\kappa}}= & \frac{\tilde{h}_{\tilde{\kappa}}}{\tilde{p}_{\tilde{\kappa}}}\left\|\varphi-\mathcal{L}^{*} \tilde{z}_{\mathrm{DG}}\right\|_{L_{2}(\tilde{\kappa})} \\
& +\left(\frac{\tilde{h}_{\tilde{\kappa}}}{\tilde{p}_{\tilde{\kappa}}}\right)^{1 / 2}\left(\left\|\left[\tilde{z}_{\mathrm{DG}}\right]\right\|_{\partial_{+} \tilde{\kappa} \backslash \Gamma_{+}}+\left\|\chi-\tilde{z}_{\mathrm{DG}}\right\|_{\partial_{+} \tilde{\kappa} \cap \Gamma_{+}}\right)
\end{aligned}
$$

for

$$
\mathcal{E}_{\mathrm{D}} \equiv\left|\mathcal{E}_{\Omega}\left(u_{\mathrm{DG}}, h, p, z\right)-\mathcal{E}_{\Omega}\left(u_{\mathrm{DG}}, h, p, \tilde{z}_{\mathrm{DG}}\right)\right| .
$$

The error indicator (4.7) arises from a Type II a posteriori error bound on $\mathcal{E}_{\mathrm{D}}$ upon setting all constants in the bound (such as the stability factor $C_{\text {stab }}$ of the dualdual problem and the interpolation constant $C_{\text {int }}$ ) to unity; cf. Theorem 5 and the 
subsequent Remark 6 with $m=1$, and note that $\Phi_{1}\left(p_{\tilde{\kappa}}, 1\right)$ is $\mathcal{O}(1)$ by $(3.26)$. The $h p-$ adaptive algorithm for the dual problem will be based on the fixed fraction strategy outlined by Rannacher [16]. Consequently, the absolute size of $\eta_{\tilde{\kappa}}\left(\tilde{p}_{\tilde{\kappa}}\right)$ is insignificant: only the relative sizes of these quantities matter; in particular, this justifies setting all constants to unity in the dual error indicator (4.7). Once the elements have been flagged for refinement/derefinement, $\tilde{h}_{\tilde{\kappa}}$ and $\tilde{p}_{\tilde{\kappa}}$ are altered accordingly by estimating the local regularity $\tilde{l}_{\tilde{\kappa}}$ of the dual solution on the dual mesh $\tilde{\mathcal{T}}$ as above by calculating $\eta_{\tilde{\kappa}}$ using a $\tilde{p}_{\tilde{\kappa}}$ and $\tilde{p}_{\tilde{\kappa}}-1$ representation of $\tilde{z}_{\mathrm{DG}}$, together with the a priori error bound (3.29).

\section{$5 \quad$ Numerical experiments}

In this section we present a number of experiments to numerically verify the a priori error bounds derived in Section 3, as well as to demonstrate the performance of the $h p$-adaptive algorithm outlined in Section 4.2.

\subsection{Example 1}

In this example we let $\Omega=(-1,1)^{2}, \mathbf{b}=\left(2-y^{2}, 2-x\right), c=1+(1+x)(1+y)^{2}$ and $f$ is chosen so that the analytical solution to (2.1) is given by

$$
u(x, y)=1+\sin \left(\pi(1+x)(1+y)^{2} / 8\right)
$$

cf. [13]. Furthermore, we choose the functional of interest $J(\cdot)$ to represent the mean flow of $u$ over $\Omega$, i.e. $J(\cdot) \equiv M_{\psi}(\cdot)$, where $M_{\psi}(\cdot)$ is given by (3.2); here, we define the weight function $\psi$ so that the solution of the corresponding dual problem (3.3) is given by

$$
z=4 \sin (\pi(1+x) / 2) \sin (\pi(1+y) / 2) \mathrm{e}^{-(2+x+y)^{2} / 2} .
$$

Thus, the true value of the mean flow of $u$ over $\Omega$ is $M_{\psi}(u)=3.9381$.

Here, we investigate the asymptotic behaviour of the $h p$-DGFEM on a sequence of successively finer square and quadrilateral meshes for different $p$. In each case the quadrilateral mesh is constructed from a uniform $N \times N$ square mesh by randomly perturbing each of the interior nodes by up to $10 \%$ of the local mesh size, cf. [14].

In Figure 1 we present a comparison of the error in the functional $\left|J(u)-J\left(u_{\mathrm{DG}}\right)\right|$ with the mesh size $h$ for $p=1,2,3$. Here, we observe that $\left|J(u)-J\left(u_{\mathrm{DG}}\right)\right|$ converges to zero at the rate $\mathcal{O}\left(h^{2 p+1}\right)$ as the mesh is refined for each fixed $p$. Thereby, confirming Theorem 11 in the case when the assumptions on the data (3.19) are violated, cf. Remark 7. Finally, we investigate the convergence of the $h p$-DGFEM with $p$-enrichment for fixed $h$. Since the true solution (5.1) is a (real) analytic function, we expect to observe exponential rates of convergence, cf. Section 3. Indeed, Figure 2 clearly illustrates this behaviour: on the linear-log scale, the convergence plots for each $p$ become straight lines as the degree of the approximating polynomial is increased. Furthermore, we observe from Figures $1 \& 2$ that the $h$-and $p$-convergence, respectively, of the $h p-\mathrm{DGFEM}$ is robust with respect to mesh distortion. 


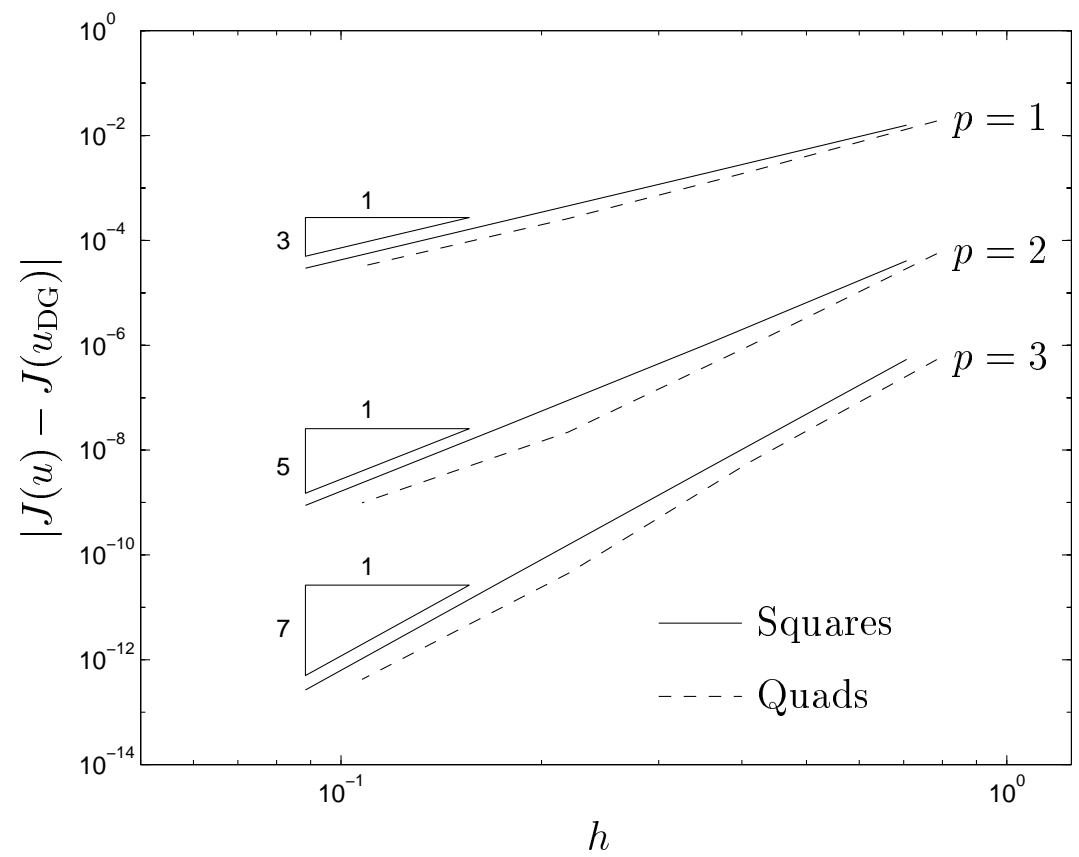

Figure 1: Example 1. Convergence of the $h p$-DGFEM with $h$-refinement.

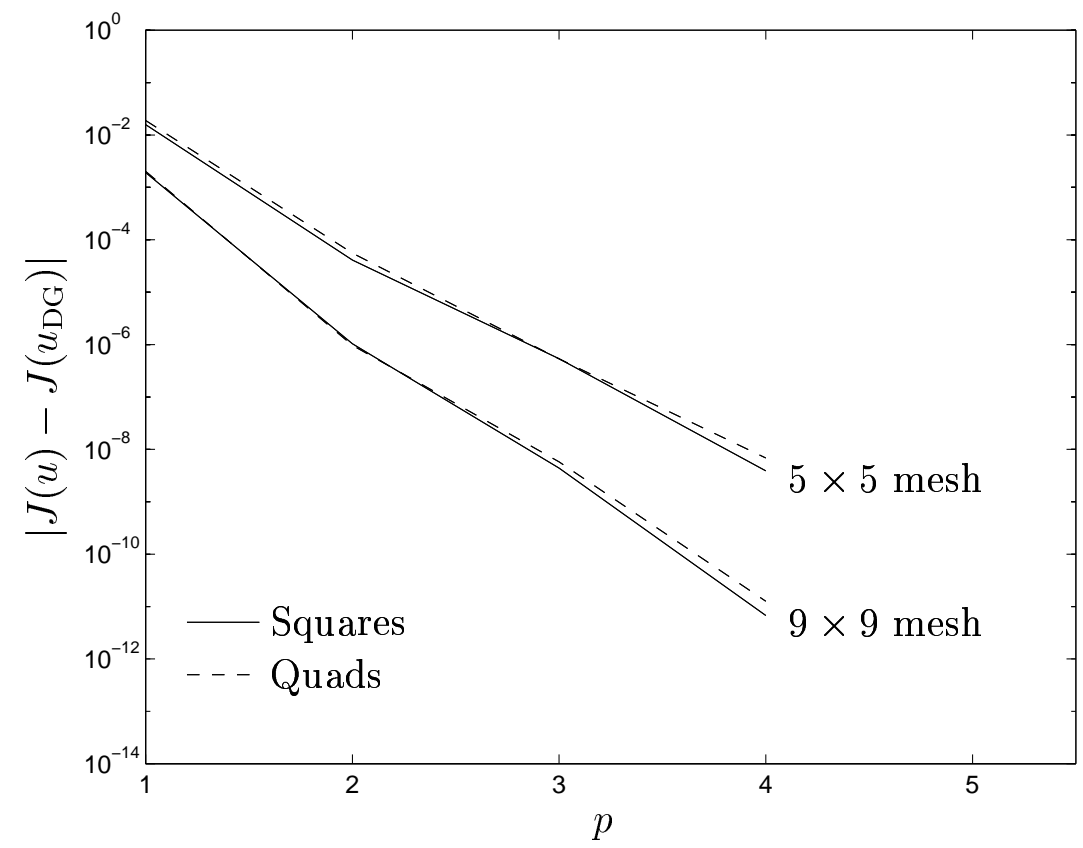

Figure 2: Example 1. Convergence of the $h p$-DGFEM with $p$-refinement. 


\begin{tabular}{||c|c|c|c|c|c|c||}
\hline Mesh & DOF & $\left|N_{\psi}\left(u-u_{\mathrm{DG}}\right)\right|$ & $\mathcal{E}_{\mathrm{P}}$ & $\mathcal{E}_{\mathrm{D}}$ & $\theta$ & $\mathcal{E}_{\mathrm{P}} / \mathcal{E}_{\mathrm{D}}$ \\
\hline 1 & 16 & $6.112 \times 10^{-2}$ & $2.964 \times 10^{-2}$ & $5.707 \times 10^{-2}$ & 0.48 & 0.52 \\
\hline 2 & 36 & $8.763 \times 10^{-2}$ & $6.169 \times 10^{-2}$ & $2.807 \times 10^{-2}$ & 0.70 & 2.20 \\
\hline 3 & 90 & $3.547 \times 10^{-2}$ & $3.201 \times 10^{-2}$ & $4.803 \times 10^{-3}$ & 0.90 & 6.66 \\
\hline 4 & 280 & $6.036 \times 10^{-3}$ & $5.496 \times 10^{-3}$ & $1.603 \times 10^{-3}$ & 0.91 & 3.43 \\
\hline 5 & 702 & $1.472 \times 10^{-3}$ & $1.811 \times 10^{-3}$ & $1.158 \times 10^{-4}$ & 1.23 & 15.64 \\
\hline 6 & 1904 & $4.107 \times 10^{-5}$ & $2.482 \times 10^{-4}$ & $3.941 \times 10^{-6}$ & 6.04 & 62.98 \\
\hline 7 & 3679 & $2.502 \times 10^{-5}$ & $3.506 \times 10^{-5}$ & $3.353 \times 10^{-6}$ & 1.40 & 10.46 \\
\hline 8 & 6414 & $5.715 \times 10^{-7}$ & $2.675 \times 10^{-6}$ & $3.320 \times 10^{-7}$ & 4.68 & 8.06 \\
\hline 9 & 10493 & $9.012 \times 10^{-8}$ & $4.524 \times 10^{-7}$ & $2.083 \times 10^{-8}$ & 5.02 & 21.72 \\
\hline 10 & 14107 & $3.175 \times 10^{-8}$ & $1.092 \times 10^{-7}$ & $9.175 \times 10^{-9}$ & 3.43 & 11.90 \\
\hline
\end{tabular}

Table 1: Example 2. $h p$-mesh refinement algorithm.

\subsection{Example 2}

Here we consider a compressible hyperbolic problem subject to discontinuous inflow boundary condition, with $\mathbf{b}=\left(2 y^{2}-4 x+1,1+y\right), c=0$ and $f=0$. The characteristics enter the computational domain $\Omega$ from three sides of $\Gamma$, namely from $x=0, y=0$ and $x=1$, and exit $\Omega$ through $y=1$. Thus, we may prescribe

$$
u(x, y)= \begin{cases}0 & \text { for } x=0,0.5<y \leq 1 \\ 1 & \text { for } x=0,0 \leq y \leq 0.5 \\ 1 & \text { for } 0 \leq x \leq 0.75, y=0 \\ 0 & \text { for } 0.75<x \leq 1, y=0 \\ \sin ^{2}(\pi y) & \text { for } x=1,0 \leq y \leq 1\end{cases}
$$

In this example we choose the functional of interest $J(\cdot)$ to represent the normal flux through the outflow boundary $\Gamma_{+}$, i.e. $J(\cdot) \equiv N_{\psi}(\cdot)$, where $N_{\psi}(\cdot)$ is given by (3.4); here, we define the weight function $\psi$ by

$$
\psi=2+\arctan ((x-1 / 2) / \varepsilon) \text { for } 0 \leq x \leq 1, y=1,
$$

where $\varepsilon=0.02$. Thereby, the true value of the outward normal flux is $N_{\psi}(u)=$ 2.0203. The analytical solutions to both the primal and dual problems are shown in Figures 3(a) \& 3(b), respectively. Furthermore, to understand how the terms in the a posteriori error bound (3.14) interact with each other, in Figures 3(c) \& $3(\mathrm{~d})$, we have plotted the $L_{2}(\kappa)$ norm of the internal residual $r_{h, p}$ and the exact weight function $z-z_{h, p}$ on a $65 \times 65$ mesh with $p=1$. Here, we observe that $\left\|r_{h, p}\right\|_{L_{2}(\kappa)}$ is large in the vicinity of the discontinuities as we would expect, while the weight function $\left\|z-z_{h, p}\right\|_{L_{2}(\kappa)}$ is large in region where the layer in $\psi$ enters the computational domain through $\Gamma_{+}$. The product of these two quantities is shown in Figure 3(e); here, we observe that the discontinuity emanating from $(x, y)=(0,0.5)$ will have very little effect on the error in the linear function $N_{\psi}(\cdot)$ (see below). 


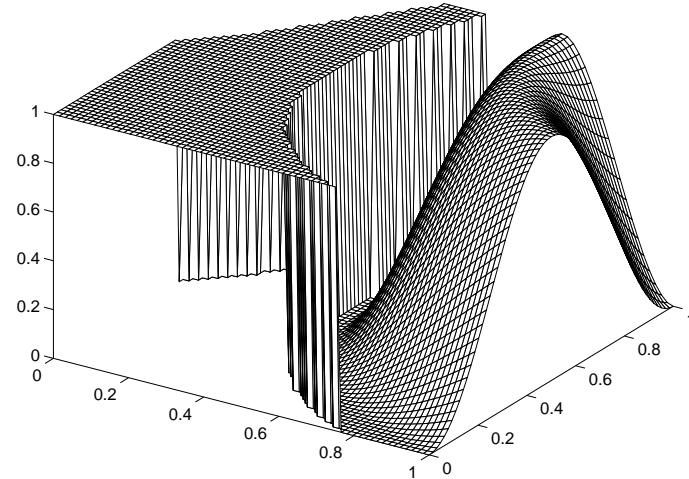

(a)

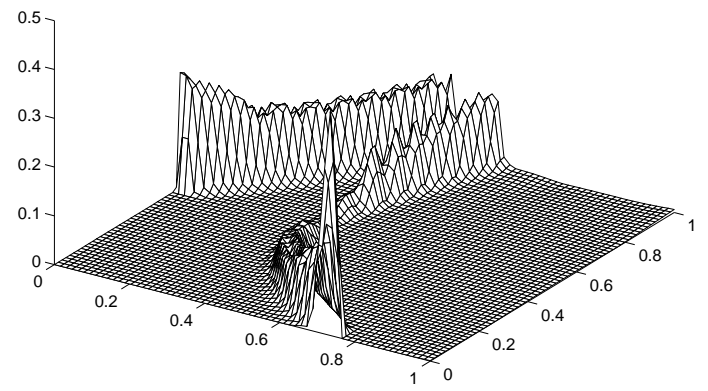

(c)

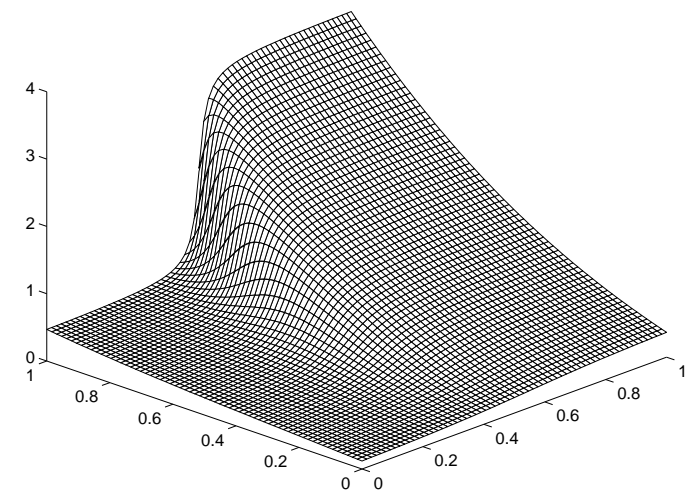

(b)

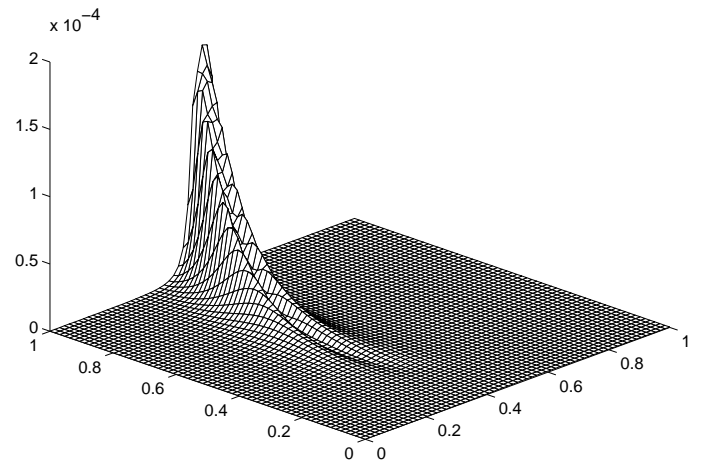

(d)



(e)

Figure 3: Example 2. (a) Analytical solution to the primal problem; (b) Analytical solution to the dual problem; (c) Internal residual term $\left\|r_{h, p}\right\|_{L_{2}(\kappa)}$ on a $65 \times 65$ mesh with $p=1$; (d) Weighting term $\left\|z-z_{h, p}\right\|_{L_{2}(\kappa)}$ on a $65 \times 65$ mesh with $p=1$; (e) Product of (c) \& (d), i.e. $\left\|r_{h, p}\right\|_{L_{2}(\kappa)}\left\|z-z_{h, p}\right\|_{L_{2}(\kappa)}$ on a $65 \times 65$ mesh with $p=1$. 


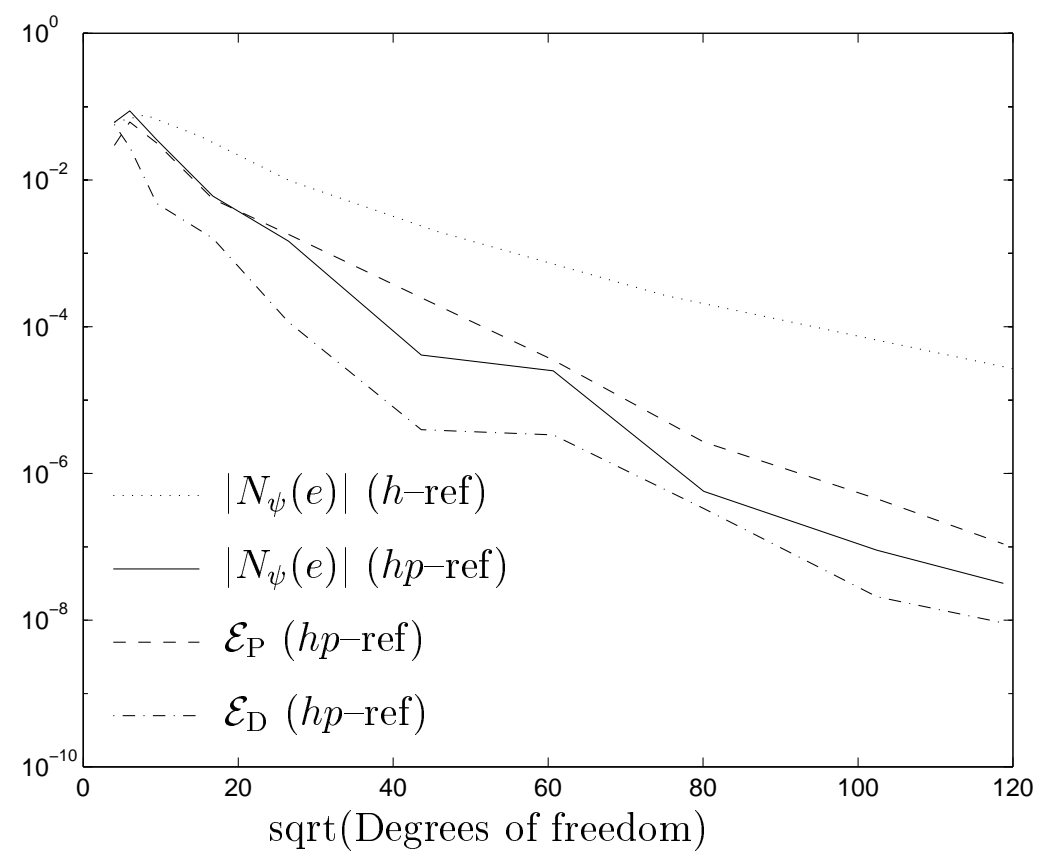

Figure 4: Example 2. Comparison between $h$ - and $h p$-adaptive mesh refinement.

In Table 1 we show the performance of the adaptive algorithm presented in Section 4.2 for TOL $=10^{-7}$; we note that this level of accuracy may be far beyond what is of practical importance, but is chosen to illustrate that the true error and the bound $\mathcal{E}_{\mathrm{P}}$ exhibit the same asymptotic behaviour as the finite element space $S^{\mathbf{p}}(\Omega, \mathcal{T}, \mathbf{F})$ is enriched. In Table 1 we show the mesh number, the number of degrees of freedom (DOF) in $S^{\mathbf{p}}(\Omega, \mathcal{T}, \mathbf{F})$, the true error in the functional $\left|N_{\psi}\left(u-u_{\mathrm{DG}}\right)\right|$, the error bound $\mathcal{E}_{\mathrm{P}}$, the remaining error $\mathcal{E}_{\mathrm{D}}$ (which in general will be non-computable), the effectivity index $\theta=\mathcal{E}_{\mathrm{P}} /\left|N_{\psi}\left(u-u_{\mathrm{DG}}\right)\right|$ and the ratio of $\mathcal{E}_{\mathrm{P}}$ and $\mathcal{E}_{\mathrm{D}}$. Here, we see that initially on very coarse meshes $\mathcal{E}_{\mathrm{P}}$ slightly underestimates the true error in the functional; however, as the finite element space is enriched the error bound overestimates $\left|N_{\psi}(u)-N_{\psi}\left(u_{\mathrm{DG}}\right)\right|$ by a consistent factor in the range 1-6. Furthermore, we see that the remaining error term $\mathcal{E}_{\mathrm{D}}$ is about an order of magnitude smaller than the computable part of the a posteriori error bound $\mathcal{E}_{\mathrm{P}}$; this numerically justifies neglecting this term in the construction of the stopping criterion (4.4) for the design of our adaptive algorithm for the primal problem.

In Figure 4 we plot the results shown in Table 1; in particular, we plot $\mid N_{\psi}(u)-$ $N_{\psi}\left(u_{\mathrm{DG}}\right) \mid, \mathcal{E}_{\mathrm{P}}$ and $\mathcal{E}_{\mathrm{D}}$ using $h p$-refinement against the square-root of the number of degrees of freedom on a linear-log scale. We see that after the initial transient, the error in the computed functional using $h p$-refinement becomes a straight line, thereby indicating exponential convergence, cf. Remark 12. Furthermore, in Figure 4 we plot the true error in the linear functional using $h$-refinement; here, we clearly observe the superiority of the adaptive $h p$-refinement algorithm. Indeed, on the final mesh the true error in the linear functional using $h p$-refinement is almost 3 orders 
of magnitude smaller than the true error in $N_{\psi}(\cdot)$ when $h$-refinement is employed.

Finally, in Figures $5 \& 6$ we show the primal and dual meshes after 6 and 9 adaptive mesh refinements, respectively. For clarity, in each case we show the $h^{-}$ mesh alone, as well as the corresponding distribution of the polynomial degree and the percentage of elements with that degree. From Figure 5, we see that the elements in the primal mesh have been refined along the first discontinuity emanating from $(x, y)=(0.75,0)$, since the dual solution has a layer in this region as well. In contrast, elements lying on the second discontinuity in the primal problem, which emanates from $(x, y)=(0,0.5)$ have been less refined since the dual solution is smooth here and hence the corresponding weights involving $\tilde{z}_{\mathrm{DG}}-z_{h, p}$ are inactive, cf. Figure 3(e). Furthermore, the mesh for the dual solution is concentrated within the steep layer in the weight function $\psi$; the inherent smoothing in the dual problem introduced by the compressible nature of $\mathbf{b}$ leads to $p$ refinement in this layer as the flow moves away from $\Gamma_{+}$. The same behaviour is observed in Figure 6 for the primal and dual solutions.

\subsection{Example 3}

In this final example, we consider the same primal problem presented in Section 5.2. Furthermore, we again let $J(\cdot)$ denote the normal flux through the outflow boundary $\Gamma_{+}$, i.e. $J(\cdot) \equiv N_{\psi}(\cdot)$, where $N_{\psi}(\cdot)$ is given by $(3.4)$; however, here we define the weight function $\psi$ by

$$
\psi= \begin{cases}1+\sin (2 \pi(4 x-1)) & \text { for } 1 / 4 \leq x \leq 3 / 4 \\ 0 & \text { otherwise }\end{cases}
$$

In this case, the true value of the outward normal flux is $N_{\psi}(u)=0.9175$. In Figures $7(\mathrm{a}), 7(\mathrm{~b})$ and $7(\mathrm{c})$ we plot the analytical to the dual problem, the weight function $\left\|z-z_{h, p}\right\|_{L_{2}(\kappa)}$ and the product of the weight function $\left\|z-z_{h, p}\right\|_{L_{2}(\kappa)}$ with the $L_{2}(\kappa)$ norm of the internal residual $r_{h, p}$ (see Figure 3(c) for the plot of $\left\|r_{h, p}\right\|_{L_{2}(\kappa)}$ ), respectively. From Figure $7(\mathrm{~b})$, we see that the weighting term $\left\|z-z_{h, p}\right\|_{L_{2}(\kappa)}$ is large in the vicinity of the discontinuities emanating from $\Gamma_{+}$. By multiplying these terms by $\left\|r_{h, p}\right\|_{L_{2}(\kappa)}$, cf. Figure $7(\mathrm{c})$, we see that the discontinuity emanating from $(0.75,1)$ will dominate the error in the outward normal flux $N_{\psi}(\cdot)$; though, there are visible peaks emanating from the top of the sin function as well as the second discontinuity located at $(0.25,1)$.

In Figure 8 we show the performance of the adaptive algorithm for TOL $=10^{-6}$. We note that since both the primal and dual solutions are not smooth, we no longer expect to observe exponential convergence of the error in the functional $N_{\psi}(\cdot)$; thus, here we plot the same quantities as in Figure 4 against the number of degrees of freedom in $S^{\mathbf{p}}(\Omega, \mathcal{T}, \mathbf{F})$ on a $\log -\log$ scale. Here, we clearly observe that the error bound $\mathcal{E}_{\mathrm{P}}$ over estimates the true error by a consistent factor between 1-10. Furthermore, as in the previous example, the remaining error term $\mathcal{E}_{\mathrm{D}}$ is about an order of magnitude smaller than $\mathcal{E}_{\mathrm{P}}$, thereby justifying the absorption of $\mathcal{E}_{D}$ into 
$\mathcal{E}_{\mathrm{P}}$. In addition, in Figure 8 we plot the true error in the linear functional using $h-$ refinement; here, we observe that the true error in $N_{\psi}(\cdot)$ is (almost) always smaller than if $h p$-refinement is employed. Indeed, on the final mesh the true error in $N_{\psi}(\cdot)$ is almost 2 orders of magnitude smaller if $h p$-refinement is employed as opposed to $h$-refinement only.

Finally, in Figures $9 \& 10$ we show the primal and dual meshes after 6 and 9 adaptive mesh refinements, respectively. From Figure 9, we see that the primal mesh has only been $h$-refined in a small neighbourhood of the two discontinuities in $u$ as they exit the computational domain $\Omega$. Furthermore, from Figure 10, we now see that the $h-$ mesh has been refined in the vicinity of the discontinuity emanating from $(x, y)=(0,0.5)$, while the $h$-mesh has in fact been coarsened in the region containing the second discontinuity emanating from $(x, y)=(0.5,0)$. In this latter region, the local polynomial degree has been enriched as the primal solution $u$ is smooth here. Finally, we note that Figures $9 \& 10$ show that the dual mesh has been extensively $h$-refined in the vicinity of both discontinuities, with the degree $p$ of the approximating polynomial increased as we move into the parts of the computational domain where the dual solution $z$ is smooth.

\section{Concluding remarks}

In this article we have developed the a posteriori error analysis of the $h p$-version of the discontinuous Galerkin finite element method. In particular, by using a hyperbolic duality argument, we have derived computable error bounds for linear functionals of the solution, such as the mean flow of the field over the computational domain $\Omega$ and the normal flux through the outflow boundary $\Gamma_{+}$. Furthermore, based on our a posteriori error bound, we have designed and implemented a fully automatic adaptive algorithm that is capable of exploiting both local mesh subdivision and local polynomial-degree enrichment. Numerical experiments have been presented which clearly highlight the superiority of such a general adaptive strategy over the traditional $h$-refinement method, where the degree of the approximating polynomial $p$ is kept fixed at some low value.

\section{References}

[1] S. Adjerid, M. Aiffa, J.E. Flaherty, Computational methods for singularly perturbed systems. In J. Cronin and R.E. O'Malley, editors, Singular Perturbation Concepts of Differential Equations, AMS, Providence, 1998.

[2] M. Ainsworth And B. Senior, An adaptive refinement strategy for hp-finite element computations. Appl. Numer. Maths. 26:165-178, 1998.

[3] I. BABušKa AND M. SuRI, The hp-Version of the Finite Element Method with quasiuniform meshes. $\mathrm{M}^{2}$ AN Modél. Math. Anal. Numér. 21:199-238, 1987. 
[4] C. Bardos, Problèmes aux limites pour les équations aux dérivées partielles du premier ordre à coefficients réels; théorèmes d'approximation; application à l'équation de transport. Ann. Sci. École Norm. Sup. (4) 3:185-233, 1970.

[5] R. Becker And R. Rannacher, Weighted a posteriori error control in FE Methods, Interdisziplinäres Zentrum für Wissenschaftliches Rechnen, Universität Heidelberg, Preprint No. 1, 1996.

[6] K.S. Bey AND J.T. Oden, hp-Version discontinuous Galerkin methods for hyperbolic conservation laws. Comput. Methods Appl. Mech. Engrg. 133:259-286, 1996.

[7] D. Braess, Finite Elements. Theory, Fast Solvers, and Applications in Solid Mechanics. Cambridge University Press, 1997.

[8] B. Cockburn, G.E. Karniadakis And C.-W. Shu. The development of discontinuous Galerkin methods. In: B. Cockburn, G. Karniadakis, and C.-W. Shu (Eds.) Discontinuous Galerkin Finite Element Methods. Lecture Notes in Computational Science and Engineering, Volume 11. Springer-Verlag 2000.

[9] R. Dautray and J.-L. Lions, Mathematical Analysis and Numerical Methods for Science and Technology, Volume 6, Evolution Problems II. Springer-Verlag, 1993.

[10] W. GUi AND I. BABUŠKA, The $h, p$ and $h-p$ versions of the finite element method in 1 Dimension. Part III. The adaptive $h-p$ version. Numer. Math. 49:659-683, 1986.

[11] R. Hartmann, Adaptive FE-methods for conservation equations. The Eighth International Conference on Hyperbolic Problems. Theory, Numerics, Applications (HYP2000). Submitted for publication.

[12] P. Houston, R. Rannacher And E. SÜli, A posteriori error analysis for stabilised finite element approximations of transport problems. (To appear in Computer Methods in Applied Mechanics and Engineering, 2000).

[13] P. Houston, Ch. Schwab And E. Süli, Discontinuous hp-finite element methods for advection-diffusion problems. Numerical Analysis Group Research Report NA00/15, University of Oxford, 2000. (Submitted for publication) http://web.comlab.ox.ac.uk/oucl/publications/natr/na-00-15.html

[14] P. Houston, Ch. Schwab And E. Süli, Stabilized hp-finite element methods for first-order hyperbolic problems. SIAM J. Numer. Anal. 37(5):1618-1643, 2000.

[15] K. Erikson, D. Estep, P. Hansbo, and C. Johnson, Introduction to adaptive methods for differential equations. Acta Numerica, 105-158, 1995.

[16] R. Rannacher, Adaptive finite element methods, in H. Bulgak and C. Zenger, eds., Proc. NATO-Summer School Error Control and Adaptivity in Scientific Computing, Kluwer, Academic Publishers, 247-278, 1998,

[17] J. RAUCH, $L_{2}$ is a continuable initial condition for Kreiss' mixed problems. Comm. Pure Appl. Math., 25:265-285, 1972. 
[18] Ch. Schwab, p- and hp-Finite Element Methods. Theory and Applications to Solid and Fluid Mechanics. Oxford University Press, 1998.

[19] E. SÜLI, A posteriori error analysis and adaptivity for finite element approximations of hyperbolic problems. In: D. Kröner, M. Ohlberger and C. Rohde (Eds.) An Introduction to Recent Developments in Theory and Numerics for Conservation Laws. Lecture Notes in Computational Science and Engineering. Volume 5, pages 123-194. Springer-Verlag, 1998.

[20] E. SÜLI, P. Houston And CH. SchwaB, hp-Finite element methods for hyperbolic problems. In: J. R. Whiteman, editor, The Mathematics of Finite Elements and Applications X. MAFELAP 1999. Elsevier, 143-162, 2000. 

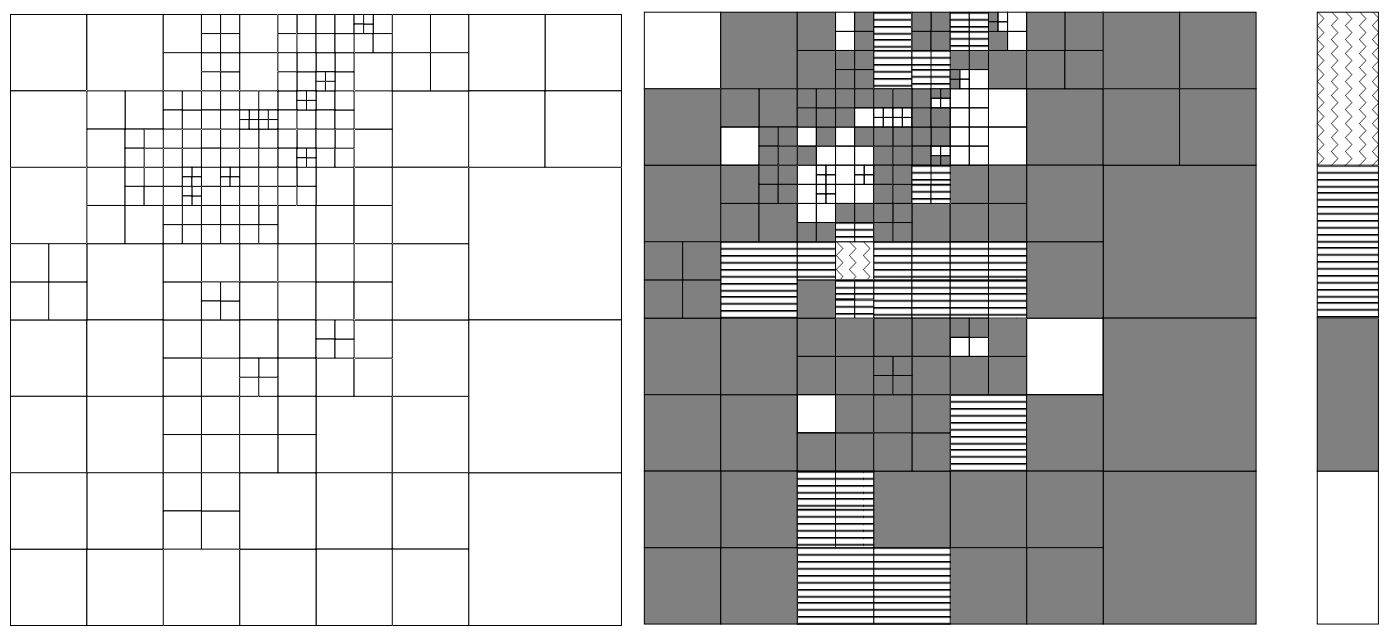

$5(1 \%)$
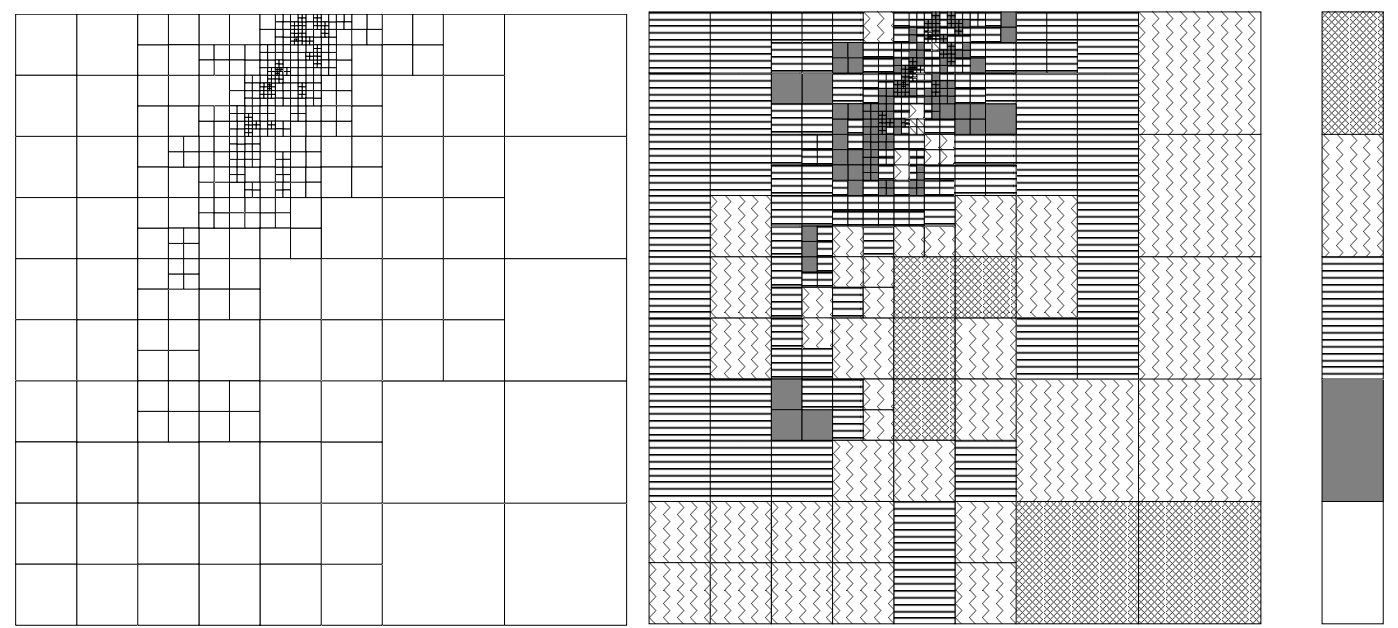

$4(16 \%)$

$3(57 \%)$

$2(26 \%)$

$5(1 \%)$

$4(10 \%)$

$3(42 \%)$

$2(44 \%)$

$1(3 \%)$

Figure 5: Example 2. Mesh 7: Primal (top: 235 elements, 303 nodes and 3679 degrees of freedom) and Dual (bottom: 493 elements, 616 nodes and 6781 degrees of freedom) $h$ - and $h p$-meshes. Here, $N_{\psi}\left(u-u_{\mathrm{DG}}\right)=2.502 \times 10^{-5}, \mathcal{E}_{\mathrm{P}}=3.506 \times 10^{-5}$ and $\theta=1.40$. 

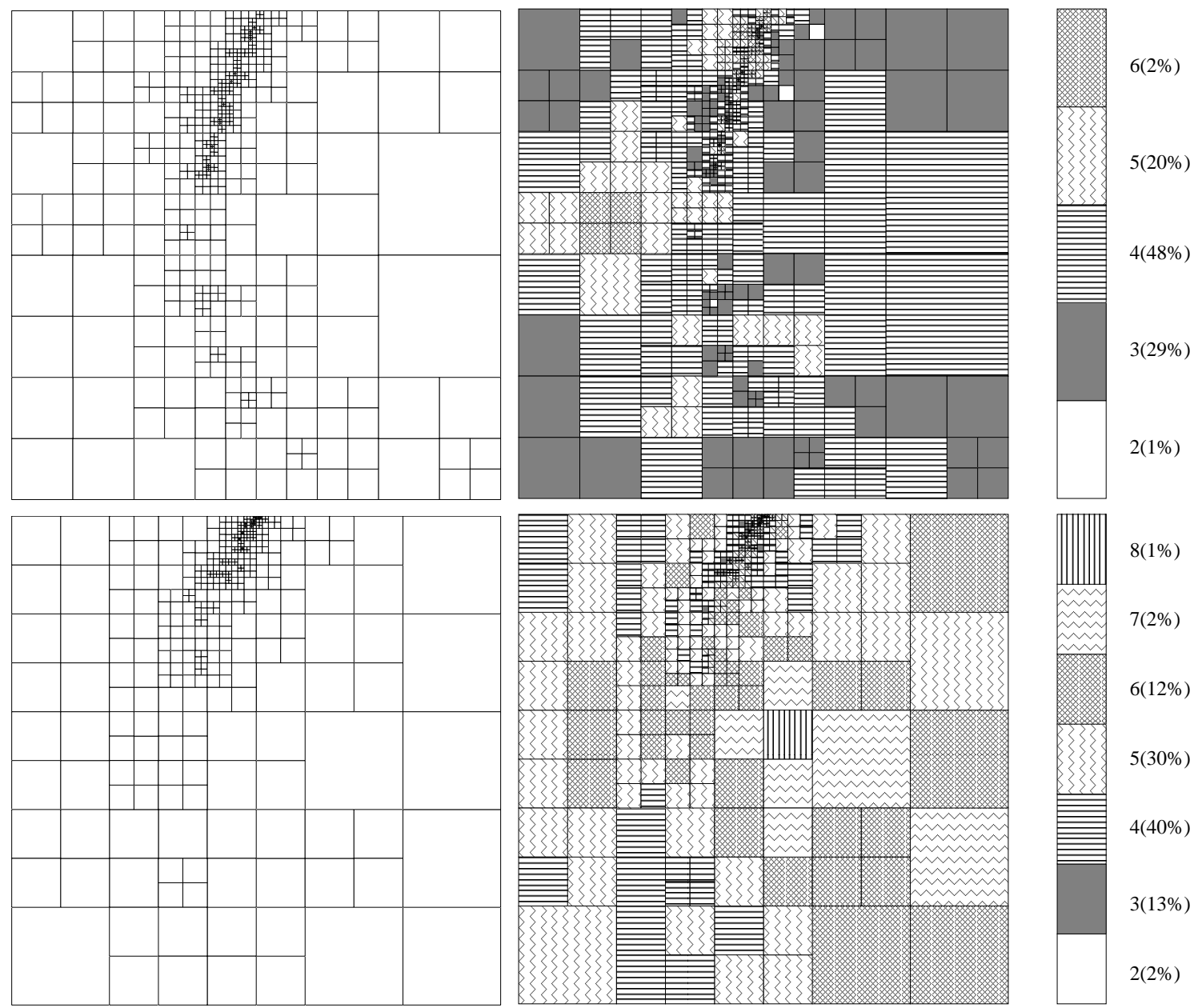

Figure 6: Example 2. Mesh 10: Primal (top: 565 elements, 719 nodes and 14107 degrees of freedom) and Dual (bottom: 445 elements, 564 nodes and 13629 degrees of freedom) $h-$ and $h p-$ meshes. Here, $N_{\psi}\left(u-u_{\mathrm{DG}}\right)=3.175 \times 10^{-8}, \mathcal{E}_{\mathrm{P}}=1.092 \times 10^{-7}$ and $\theta=3.43$. 


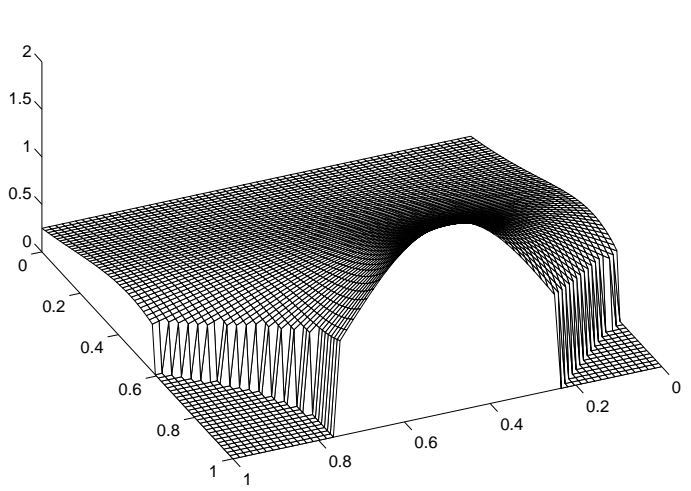

(a)

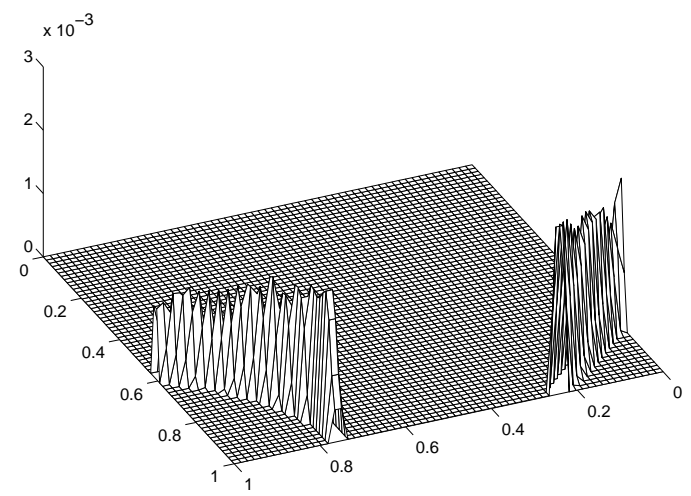

(b)

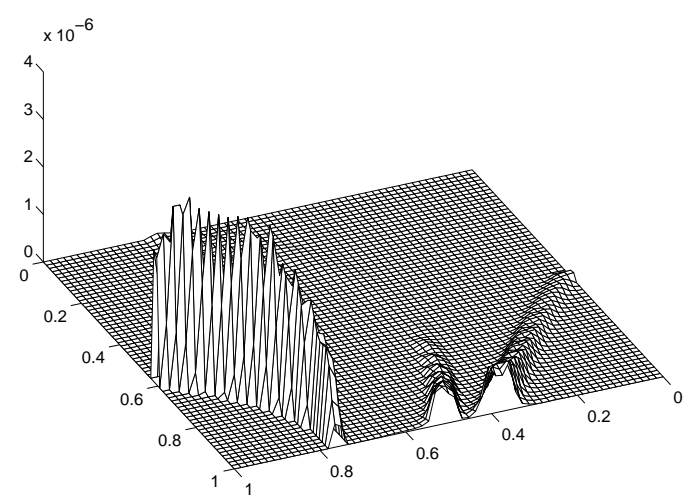

(c)

Figure 7: Example 3. (a) Analytical solution to the dual problem; (b) Weighting term $\left\|z-z_{h, p}\right\|_{L_{2}(\kappa)}$ on a $65 \times 65$ mesh with $p=1$; (c) Product of (b) \& Figure 3(c), i.e. $\left\|r_{h, p}\right\|_{L_{2}(\kappa)}\left\|z-z_{h, p}\right\|_{L_{2}(\kappa)}$ on a $65 \times 65$ mesh with $p=1$. 




Figure 8: Example 3. Comparison between $h$-and $h p$-adaptive mesh refinement. 

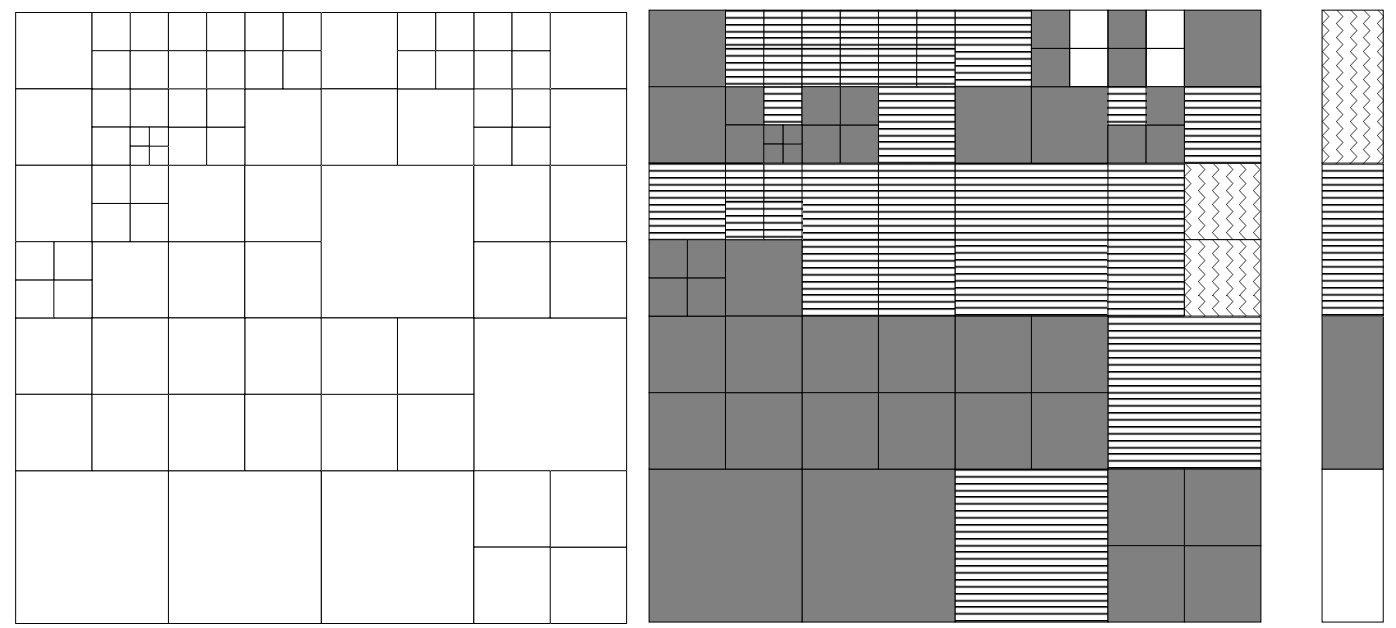

$5(2 \%)$
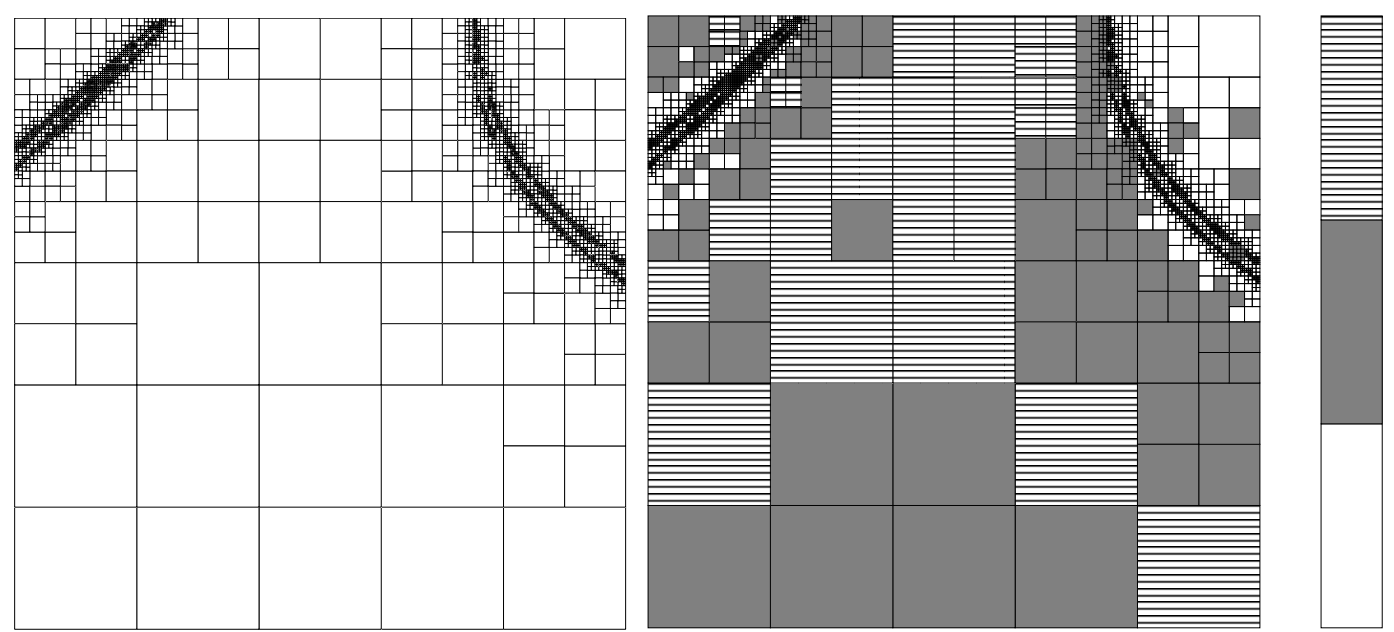

$3(2 \%)$

$2(10 \%)$

$1(88 \%)$

Figure 9: Example 3. Mesh 7: Primal (top: 82 elements, 116 nodes and 1603 degrees of freedom) and Dual (bottom: 2629 elements, 3167 nodes and 12316 degrees of freedom) $h$ - and $h p-$ meshes. Here, $N_{\psi}\left(u-u_{\mathrm{DG}}\right)=3.568 \times 10^{-6}, \mathcal{E}_{\mathrm{P}}=6.164 \times 10^{-6}$ and $\theta=1.73$. 

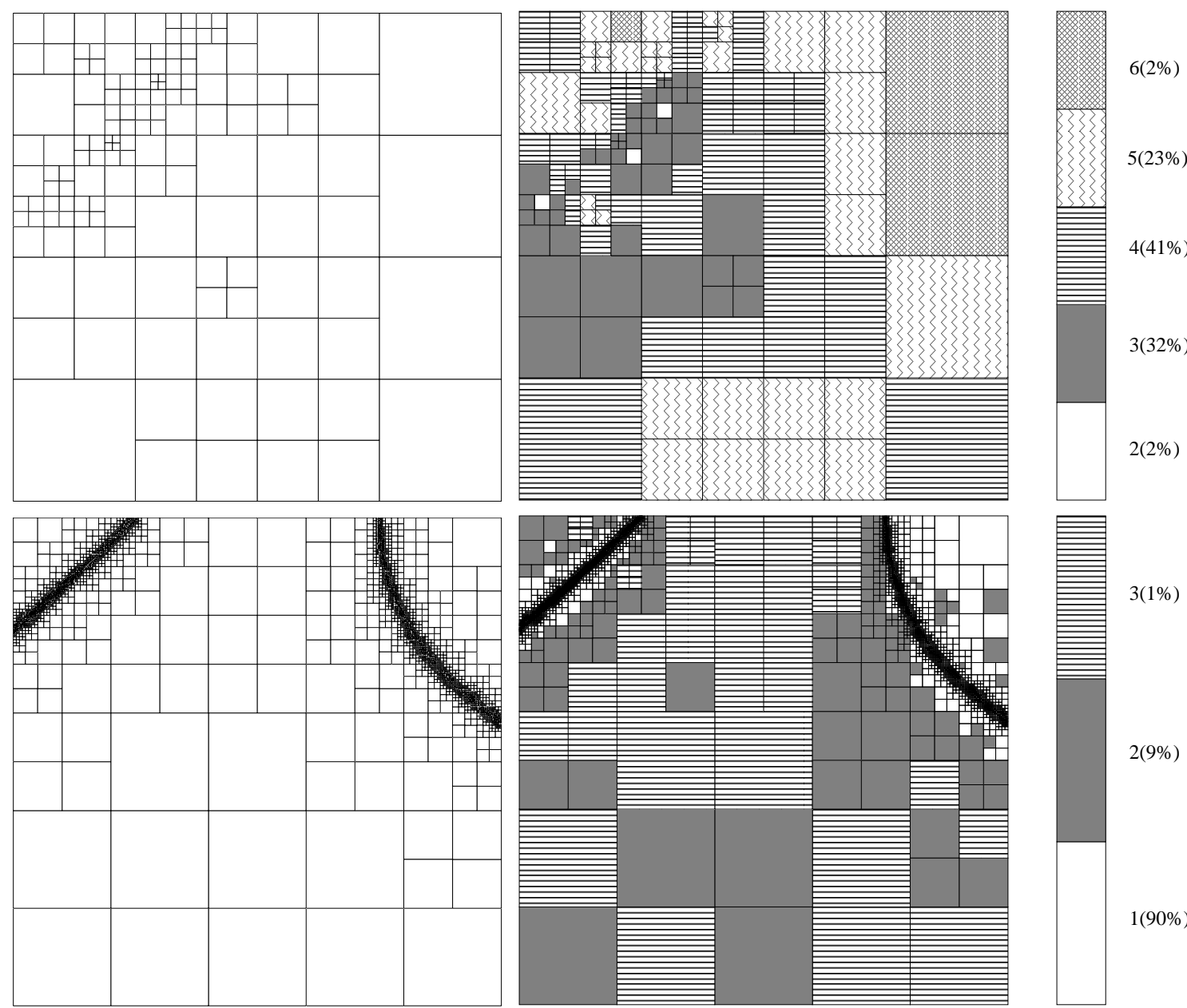

$5(23 \%)$

$4(41 \%)$

$3(32 \%)$

$2(2 \%)$

$3(1 \%)$

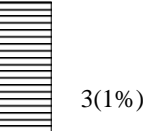

$2(9 \%)$

1(90\%)

Figure 10: Example 3. Mesh 10: Primal (top: 145 elements, 194 nodes and 3609 degrees of freedom) and Dual (bottom: 7630 elements, 9280 nodes and 34451 degrees of freedom) $h$ - and $h p$-meshes. Here, $N_{\psi}\left(u-u_{\mathrm{DG}}\right)=1.072 \times 10^{-7}, \mathcal{E}_{\mathrm{P}}=5.505 \times 10^{-7}$ and $\theta=5.14$. 